\title{
Socio-economic factors associated with an increase in fruit and vegetable consumption: a 12-year study in women from the E3N-EPIC study
}

\author{
Aurélie Affret ${ }^{1}$, Gianluca Severi ${ }^{1,2,3}$, Courtney Dow ${ }^{1}$, Francesca Romana Mancini ${ }^{1}$, \\ Grégoire Rey ${ }^{4}$, Cyrille Delpierre ${ }^{5}$, Françoise Clavel-Chapelon ${ }^{1}$, \\ Marie-Christine Boutron-Ruault ${ }^{1}$ and Guy Fagherazzi ${ }^{1, *}$ \\ ${ }^{1}$ Centre for Research in Epidemiology and Population Health (CESP), INSERM (Institut National de la Santé et de la \\ Recherche Médicale) U1018, Facultés de médecine Universités Paris-Sud, UVSQ, Université Paris-Saclay, Gustave \\ Roussy Institute, 114 rue Edouard Vaillant, 94805 Villejuif Cedex, France: ${ }^{2}$ Human Genetics Foundation (HuGeF), \\ Torino, Italy: ${ }^{3}$ Centre for Epidemiology and Biostatistics, The University of Melbourne, Melbourne, Australia: ${ }^{4}$ Inserm, \\ CépiDc, Le Kremlin-Bicêtre, France: ${ }^{5}$ Inserm U1027, Université Toulouse III, Toulouse, France
}

Submitted 8 May 2017: Final revision received 16 August 2017: Accepted 25 September 2017: First published online 29 November 2017

\begin{abstract}
Objective: To identify individual and contextual socio-economic factors associated with an increase in fruit and vegetable $(\mathrm{F} \& \mathrm{~V})$ consumption over a 12-year period and evaluate if some socio-economic factors were differentially associated with the change in consumption of some types of $F \& V$.

Design: Associations between increased F\&V consumption and socio-economic factors were studied with multivariate logistic regression.

Setting: E3N, a French prospective cohort study of 98995 women.

Subjects: E3N participants ( $n$ 58193) with information on diet in 1993 and 2005, and numerous individual and contextual socio-economic factors available.

Results: Associations between some individual socio-economic factors and changes in $\mathrm{F} \& \mathrm{~V}$ consumption were observed. For instance, women who lived in a large household ( $>3$ children $v$. no child) had higher probability of increasing their vegetable consumption $(\mathrm{OR}=1.33$; $95 \% \mathrm{CI} 1 \cdot 24,1.42)$. This association was driven by higher consumption of courgette and raw cucumber. Living with a partner was associated with higher odds of increasing consumption of fruits (OR $=1.07 ; 95 \%$ CI 1.02, 1.13) such as pear, peach and grape.

Conclusions: Certain individual socio-economic factors, but none of the contextual socio-economic factors examined, were associated with an increase in F\&V consumption. Factors associated with an increase in total $\mathrm{F} \& \mathrm{~V}$ consumption were not necessarily associated with an increase in fruit or vegetable consumption separately, or with an increase in each subtype of fruit or vegetable. Magnitudes of the different associations observed also differed when F\&V were considered together, separately or by subtype. Increases in $\mathrm{F} \& \mathrm{~V}$ consumption were mostly observed in women with high socio-economic position. To develop effective nutritional interventions and policies that take the socio-economic environment of individuals into account, we recommend future research to further focus on (i) pathways through which population characteristics might influence changes in F\&V consumption and (ii) existing interactions between individual and contextual socio-economic factors.
\end{abstract}

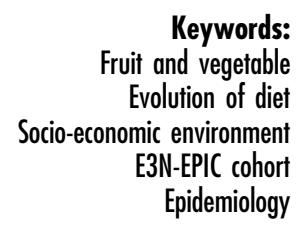

Keywords:

ruit and vegetable

Evolution of diet

E3N-EPIC cohort

Epidemiology
Consumption of fruits and vegetables is associated with a reduced risk of obesity ${ }^{(1)}, \mathrm{CVD}^{(2)}$ and some cancers ${ }^{(3)}$, as well as a lower risk of all-cause and cardiovascular mortality ${ }^{(4)}$. The protective effect of fruits and vegetables is attributed to their fibre content, essential micronutrients and non-nutritive phytochemicals $^{(5)}$. International agencies ${ }^{(6,7)}$ and several countries ${ }^{(8-11)}$ currently recommend people to consume more than five servings daily, disregarding the types of fruit or vegetable. 
It is well established that dietary habits including fruit and vegetable consumption are strongly patterned by socio-economic status ${ }^{(12-16)}$, with socio-economically disadvantaged groups less likely to consume fruits and vegetables ${ }^{(17-19)}$. The socio-economic environment of individuals is complex and multifactorial. Nevertheless, its influence has generally been studied by considering single specific and conventional socio-economic factors ${ }^{(20)}$ such as education $^{(21)}$ or income $\operatorname{tax}^{(22)}$. It has been suggested that, in order to capture the multidimensional nature of the socio-economic environment, it is preferable to simultaneously consider several socio-economic factors ${ }^{(20,23)}$. Socio-economic variables are each operationally distinct, may influence health behaviours by conceptually different processes $^{(23)}$ and are not interchangeable. It is therefore recommended, when possible, to study the influence of the socio-economic environment considering various individual and contextual socio-economic factors.

The socio-economic position of an individual is likely to be the result of all existing interactions between these factors $^{(24)}$, including money and time availabilities (examples of individual socio-economic factors), as well as access to grocery stores, transportation and neighbourhood safety (examples of contextual socio-economic factors) ${ }^{(25)}$.

It is of major importance for chronic disease prevention to understand the socio-economic situations associated with having or moving to a healthier diet, since this may help health policy makers develop nutritional guidelines that consider the socio-economic disparities and specifically address the socio-economic groups that little respond to general health-promoting messages.

Fruit and vegetable consumption is often used as a proxy for a healthy diet. Indeed, diets rich in fruits and vegetables have been associated with better overall health status $^{(26-31)}$. In addition, positive messages such as enhancing fruit and vegetable consumption are better accepted than messages focused on reducing unhealthy components of the diet ${ }^{(32,33)}$, and should lead to substituting less healthy components for fruits and vegetables.

Only a few large cohort studies have multiple dietary intake measurements repeated over time, rending the study of diet evolution difficult. Even though few studies have several dietary intake measures, to our knowledge, they have not considered the impact of both individual and contextual factors on the evolution of diet.

The present study aimed to evaluate, for the first time, the socio-economic factors (both individual and contextual) associated with an increase in total fruit and vegetable consumption over a 12 -year period, in women from the large E3N-EPIC prospective cohort study. Thanks to the detailed information in the data, we also have investigated whether these socio-economic factors were associated with an increase in fruit consumption or vegetable consumption separately, or even with an increase in consumption of specific fruits or vegetables.

\section{Methods}

\section{The E3N-EPIC cohort study}

The E3N-EPIC study is a prospective cohort study of 98995 French women recruited from a national health insurance plan covering people working in the national education system $^{(34)}$. E3N (Etude Epidemiologique aupres de femmes de l'Education Nationale) is the French component of the European Prospective Investigation into Cancer and Nutrition (EPIC) ${ }^{(35)}$ and was initiated in 1990. The procedures followed were in accordance with the Helsinki Declaration of 1975 as revised in 1983 and all procedures involving human subjects were approved by the French National Commission for Computerized Data and Individual Freedom (CNIL). All women signed a letter of informed consent to allow their data to be stored, as required by the CNIL.

Participants were sent questionnaires to update healthrelated information and newly diagnosed diseases every 2 to 3 years. The average follow-up response rate is $83 \%$ (which decreases to $68 \%$ when considering food questionnaires, which are more time-consuming) and, overall, the total loss to follow-up since 1990 is below 3\%.

\section{Study population}

A total of 74522 women answered the first food questionnaire in 1993 and among them, 60380 women answered the second one in 2005. We excluded those who under- or over-reported energy intake at these two time points ( $n 1105$ and $n 1082$, respectively) as previously described ${ }^{(36)}$. These women were in the top and bottom $1 \%$ of the distribution of energy intake to BMR, computed on the basis of age, height and weight. After their exclusion, 58193 women with available data in 1993 and 2005 were included in the present study. The study selection process is reported in the online supplementary material, Supplemental Fig. 1.

\section{Dietary data}

Dietary data were self-reported in June 1993 and July 2005 using a validated diet history questionnaire ${ }^{(37)}$. The questionnaire was divided into two parts. The first part quantified consumption by frequency (eleven categories, from 'never or less than once a month' to 'seven times a week') and portion sizes per food group or food item, and was grouped by meal occasions (eight occasions from breakfast to after-dinner snacks, including occasions such as the aperitifs before lunch and dinner). The second part provided more detailed qualitative information on separate items within one food group previously reported in the first part. In total, we were able to compute quantities in grams per day for 238 food items (foods and beverages).

A score of adherence to the national dietary guidelines ${ }^{(38)}$ was derived from the dietary data as previously described $^{(39)}$. The score ranges from 0 (minimal adherence to the dietary guidelines) to 15 (maximal adherence to the dietary guidelines). 


\section{Fruit and vegetable consumption}

The national dietary guidelines' definition of the fruit and vegetable group was used in the present study ${ }^{(40)}$. The fruit group was composed of fresh fruit, fruit in syrup, fruit purée and $100 \%$ pure fruit juice. The vegetable group included cooked and raw vegetables and soup. In total, information was available for sixteen types of fresh fruits, eighteen cooked vegetables and sixteen raw vegetables. To study associations between socio-economic factors and the most consumed fruit and vegetable subtypes, we identified the five most consumed fruits in the E3N-EPIC cohort in 1993: peaches, apples, grapefruit, pears and grapes. The five most consumed cooked vegetables in the E3N-EPIC cohort in 1993 were: green beans, courgette, carrots, tomatoes and endives. The five most consumed raw vegetables in the E3N-EPIC cohort in 1993 were: tomatoes, carrots, endives, cucumbers and radishes.

\section{Standard ratio of fruit and/or vegetable consumption in 1993}

National dietary guidelines recommend consuming at least five portions of fruit and vegetables daily, which corresponds to approximately $400 \mathrm{~g} / \mathrm{d}^{(41)}$. The mean national energy intake in women is about $1800 \mathrm{kcal} / \mathrm{d}(7530 \mathrm{~kJ} / \mathrm{d})^{(42)}$. Therefore, to take the participants' energy intake into account, we used the ratio of fruit and/or vegetable intake to energy intake. Rather than looking at the crude consumption of fruits and vegetables in grams per day, which would not be the best reflection of physiological needs, we considered fruit and/or vegetable consumption as a function of energy intake as previous studies have done ${ }^{(43,44)}$. In addition, this provides a form of standardization and the ratio gives an estimate of how fruit and/or vegetable intake contribute(s) to the overall diet. For overall fruit and vegetable consumption, the reference value was $400 \mathrm{~g} / 1800 \mathrm{kcal}$ $(400 \mathrm{~g} / 7530 \mathrm{~kJ})$, i.e. $0 \cdot 22 \mathrm{~g} / \mathrm{kcal}(0.053 \mathrm{~g} / \mathrm{kJ})$. For fruit consumption and vegetable consumption considered separately, the references were $200 \mathrm{~g} / 1800 \mathrm{kcal}(200 \mathrm{~g} / 7530 \mathrm{~kJ})$, i.e. $0 \cdot 11 \mathrm{~g} / \mathrm{kcal}(0 \cdot 027 \mathrm{~g} / \mathrm{kJ})$.

\section{Increase in fruit and vegetable consumption over time}

To study the increase in fruit and/or vegetable consumption over time, we considered changes in the ratio of daily fruit and/or vegetable intake to daily energy intake between 1993 and 2005. The variable was categorized into a binary factor, where $0=$ 'stability or decrease based on the ratio between 1993 and 2005' and $1=$ 'increase in the ratio', with 0 taken as the reference category.

\section{Socio-economic information}

We considered both individual and contextual socioeconomic data in the E3N-EPIC study. The following individual socio-economic variables were available in 1993: level of education (<high school diploma, up to 2 years of university, $>2$ years of university), occupation in 1993 (currently working $v$. not working), income (corresponding to the current professional activity reported by the women in 1992 or the last one if not currently working and divided into quartiles), number of children (four categories: $0,1,2, \geq 3$ ) and marital status (single $v$. in couple). The contextual socio-economic factors available in 1993 included place of residence in 1993 (six categories: Paris and suburbs, North, West, East, Centre and South), size of the city of residence in 1993 (computed from the commune of residence - the smallest administrative unit in France - and categorized as rural, i.e. areas with fewer than 2000 inhabitants; quasi-rural, i.e. areas with 2000 to 9999 inhabitants; quasi-urban, i.e. areas with 10000 to 99999 inhabitants; and large urban, i.e. areas with 100000 or more inhabitants) and a deprivation index named FDep99 ${ }^{(45)}$ computed for each woman in 1993 from the information reported on her commune of residence. The FDep99 had been previously constructed at the commune level using four variables obtained from the 1999 population census and the tax authority's 2001 household income data ${ }^{(46)}$ : median household income, percentage of high-school graduates in the population aged 15 years or older, percentage of blue-collar workers in the active population and unemployment rate.

We considered all these factors because they have previously been found to be related to diet ${ }^{(22,47-49)}$. Additionally, we included two less explored contextual socio-economic variables as we estimated that they could help to better characterize the socio-economic status of individuals: (i) the geographical area of birth (eight categories: Paris and suburbs, North, West, East, Centre, South, Dom-Tom (overseas departments and territories of France) and Abroad); and the size of the work area ( $\leq 10000$ inhabitants and $>10000$ inhabitants).

\section{Individual characteristics}

Age, BMI (in $\mathrm{kg} / \mathrm{m}^{2}$ ), level of physical activity (in MET-h/week, where MET is metabolic equivalent of task) and smoking status (non-smoker, former smoker, current smoker) were used for adjustment because they have been shown to be associated with diet as well as with socio-economic position ${ }^{(47,50-52)}$. All these data were available in 1993.

\section{Statistical analysis}

For variables with $<5 \%$ of values missing, missing values were imputed with the median of the study population (quantitative variables) or the mode (qualitative variables). In the case of $\geq 5 \%$ of missing values, a 'missing' category was created.

Description of the population according to the standard ratio of fruit and/or vegetable consumption in 1993 Women were described in terms of individual characteristics and socio-economic factors depending on whether their ratio of fruit and vegetable consumption in 1993 (see section 'Standard ratio of fruit and/or vegetable 
consumption in $1993^{\prime}$ ) was $\geq 0 \cdot 22$ or $<0 \cdot 22$. When consumption of fruits and consumption of vegetables were studied separately, the ratio of $\geq 0 \cdot 11$ or $<0 \cdot 11$ was considered.

Individual characteristics and socio-economic factors associated with an increase in fruit and vegetable consumption over time

Multivariable logistic regression models were performed to estimate OR of increasing consumption of fruits and/or vegetables over a 12-year period as well as their 95\% CI. Factors included in the fully adjusted models were individual socio-economic factors (level of education, occupation in 1993, income, number of children and marital status), contextual socio-economic factors (place of residence in 1993, size of the city of residence in 1993, FDep99, geographical area of birth and size of the work area) and individual characteristics (age, BMI, smoking status and level of physical activity). Models were also adjusted for energy intake evolution between 1993 and 2005 (kcal/d) as well as baseline consumption of fruits and vegetables (only baseline consumption of fruits or baseline consumption of vegetables, when considered separately; g/d).

All statistical analyses were performed using the statistical software package SAS version 9.3. A $P$ value of $<0.05$ was considered statistically significant.

\section{Results}

\section{Fruit and vegetable consumption and baseline characteristics}

The main characteristics of the study population are summarized in Table 1. In comparison with women having fruit and vegetable consumption below the standard ratio, women with consumption greater than or equal to the standard ratio were older $(16.0 v .28 \cdot 1 \%$ with age $>57.2$ years), more physically active ( $45 \cdot 1 \mathrm{v} .51 .5 \%$ with physical activity $>37.9$ MET-h/week), less frequently current smokers $(16.4 v .11 .5 \%)$ and had a comparable BMI (78.7 v. $77.7 \%$ with normal BMI). In terms of individual socio-economic factors and compared with women having fruit and vegetable consumption below the standard ratio, women with consumption greater than or equal to standard ratio had similar level of education $(90 \cdot 0$ v. $89.9 \%$ with more than high school diploma), income (30.2 v. $28.5 \%$ in the highest quartile), number of children (28.2 v. $29.4 \%$ with three or more children) and marital status (84.7 v. 83.9\% living with a partner), but they were less frequently with occupation (79.1 v. 67.7\%). In terms of contextual socio-economic factors and compared with women having fruit and vegetable consumption below the standard ratio, women with consumption greater than or equal to the standard ratio were more frequently born and living in the South of France $v$. North of France (23.6 and
$28.7 \%$ v. 30.2 and $37 \cdot 1 \%$ for the South, and 18.1 and $16.8 \% v \cdot 12 \cdot 0$ and $11 \cdot 1 \%$ for the North). No differences were observed in terms of deprivation index (34.6 v. $33.8 \%$ living in the most deprived areas) and size of the agglomeration of residence (17.9 v. 18.3\% living in large urban areas). When separately considering the consumptions of fruit and vegetables, similar characteristics were observed for women with consumption greater than or equal to the standard ratio.

The main characteristics of the study population in terms of diet are summarized in the online supplementary material, Supplemental Table 1. The mean intake of fruits and vegetables was 662.5 (sD 269.9) g/d. For women having fruit and vegetable consumption below the standard ratio the mean intake was $400 \cdot 4$ (SD 136.1) g/d, while for those having consumption greater than or equal to the standard ratio the mean intake was 753.3 (sD 244.2) g/d. In comparison with women having fruit and vegetable consumption below the standard ratio, women with consumption greater than or equal to the standard ratio were more likely to consume higher quantities of foods considered as healthy such as fish (24.9) (SD 18.6) v. 29.8 $(\mathrm{SD} 22.5) \mathrm{g} / \mathrm{d}$ ) and olive oil (3.0 (sD 3.9) v. $5.4(\mathrm{sD} 6.0) \mathrm{g} / \mathrm{d}$ ), and less quantities of foods considered as unhealthy such as French fries (12.1 (sD 13.0) v. $6 \cdot 8(\mathrm{sD} 9 \cdot 2) \mathrm{g} / \mathrm{d}$ ), pizzas (25.9 (SD 25.1) v. $18 \cdot 7$ (SD 20.1) g/d) and sandwiches (15.1 (SD 26.1) v. 7.1 (SD 15.4) g/d). They had lower energy (2417.1 (sD 585.6) kcal/d (10113 (sD 2450) kJ/d) v. $2139 \cdot 7$ (SD 519.4) kcal/d (8953 (sD 2173) kJ/d)) and alcohol intakes (16.0 (SD 17.1) v. $9.9(\mathrm{SD} \mathrm{11.9)} \mathrm{g} / \mathrm{d})$, as well as higher adherence to guidelines scores (7.5 (SD 2.0) v. $9 \cdot 6$ (SD 1.9)). When separately considering the consumptions of fruits and vegetables, similar characteristics were observed for women with consumption greater than or equal to the standard ratio.

\section{Individual socio-economic factors associated with an increase in fruit and vegetable consumption}

In the fully adjusted models, higher level of education was positively associated with an increase in fruit consumption (OR $=1.09$; 95\% 1.00, 1.18 for $>2$ years of university $v$. $<$ high school diploma), especially grapefruit consumption $(\mathrm{OR}=1 \cdot 16 ; 95 \% \mathrm{CI} 1 \cdot 08,1 \cdot 25)$. It was also associated with an increase in vegetable consumption $(\mathrm{OR}=1 \cdot 17 ; 95 \% \mathrm{CI}$ $1 \cdot 08,1 \cdot 27$ ), especially cooked vegetables (e.g. $\mathrm{OR}=1 \cdot 27$; $95 \%$ CI $1.17,1.37$ for courgette consumption; Tables 2 and 3 ). The increase in vegetable consumption was 41.4 and $23.2 \mathrm{~g} / \mathrm{d}$ on average for women with a level of education $>2$ years of university and $<$ high school diploma, respectively.

Having an occupation was associated with a higher probability of increasing vegetable consumption (OR= $1.20 ; 95 \%$ CI $1.12,1.28 v$. without occupation), with an exception of endive consumption (OR $=0.99$; $95 \%$ CI 0.93, 1.06). The increase in vegetable consumption was 48.6 and $14.6 \mathrm{~g} / \mathrm{d}$ on average for women with and without an 
Table 1 Baseline individual and socio-economic characteristics of the population according to their fruit and vegetable consumption in 1993 (E3N-EPIC cohort study, $n$ 58 193)

\begin{tabular}{|c|c|c|c|c|c|c|}
\hline \multirow[b]{2}{*}{ Percentage (\%) } & \multicolumn{2}{|c|}{ Fruit and vegetables } & \multicolumn{2}{|c|}{ Fruit } & \multicolumn{2}{|c|}{ Vegetables } \\
\hline & $\begin{array}{c}\text { Women } \\
\text { consuming less } \\
\text { than the ratiot } \\
(n 14972)\end{array}$ & $\begin{array}{l}\text { Women } \\
\text { consuming the } \\
\text { ratiot or more } \\
(n 43221)\end{array}$ & $\begin{array}{c}\text { Women } \\
\text { consuming less } \\
\text { than the ratio } \\
(n 19007)\end{array}$ & $\begin{array}{l}\text { Women } \\
\text { consuming the } \\
\text { ratio } \neq \text { or more } \\
(n 39186)\end{array}$ & $\begin{array}{c}\text { Women } \\
\text { consuming less } \\
\text { than the ratio§ } \\
(n 17685)\end{array}$ & $\begin{array}{l}\text { Women } \\
\text { consuming the } \\
\text { ratio§ or more } \\
(n 40508)\end{array}$ \\
\hline \multicolumn{7}{|l|}{ Individual characteristics } \\
\hline Age (years) & \multicolumn{2}{|c|}{ 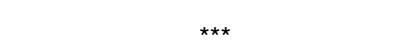 } & \multicolumn{2}{|c|}{ *** } & \multicolumn{2}{|c|}{ *** } \\
\hline$\leq 47 \cdot 1$ & 33.4 & $22 \cdot 1$ & $31 \cdot 4$ & 21.9 & $31 \cdot 0$ & $22 \cdot 4$ \\
\hline $47 \cdot 1-51 \cdot 5$ & $28 \cdot 8$ & 23.7 & $27 \cdot 9$ & 23.6 & $27 \cdot 5$ & 23.9 \\
\hline $51.5-57.2$ & $21 \cdot 8$ & $26 \cdot 1$ & 22.5 & $26 \cdot 2$ & $22 \cdot 7$ & $26 \cdot 0$ \\
\hline$>57.2$ & $16 \cdot 0$ & $28 \cdot 1$ & $18 \cdot 2$ & $28 \cdot 3$ & $18 \cdot 8$ & $27 \cdot 7$ \\
\hline BMI $\left(\mathrm{kg} / \mathrm{m}^{2}\right)$ & \multicolumn{2}{|c|}{$\star \star \star \star ~$} & \multicolumn{2}{|c|}{ ** } & \multicolumn{2}{|c|}{$\star \star \star \star ~$} \\
\hline$<18.5$ & 3.8 & $3 \cdot 1$ & $3 \cdot 4$ & $3 \cdot 2$ & $4 \cdot 1$ & $2 \cdot 9$ \\
\hline $18 \cdot 5-25$ & 78.7 & $77 \cdot 7$ & 77.9 & 78.0 & $79 \cdot 3$ & 77.4 \\
\hline $25-30$ & $14 \cdot 2$ & $16 \cdot 1$ & $15 \cdot 2$ & $15 \cdot 8$ & 13.6 & $16 \cdot 5$ \\
\hline$\geq 30$ & $3 \cdot 3$ & $3 \cdot 1$ & 3.5 & 3.0 & $3 \cdot 0$ & $3 \cdot 2$ \\
\hline Physical activity (MET-h/week) & \multicolumn{2}{|c|}{ 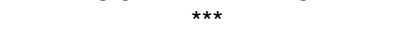 } & \multicolumn{2}{|c|}{ 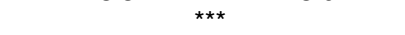 } & \multicolumn{2}{|c|}{$\star \star \star \star ~$} \\
\hline$\leq 23.1$ & $29 \cdot 3$ & $23 \cdot 6$ & 28.4 & $23 \cdot 4$ & $28 \cdot 0$ & 23.8 \\
\hline $23 \cdot 1-37 \cdot 9$ & $25 \cdot 6$ & 24.9 & $25 \cdot 5$ & 24.8 & $25 \cdot 1$ & $25 \cdot 0$ \\
\hline $37.9-60.6$ & 23.7 & $25 \cdot 3$ & 23.8 & $25 \cdot 5$ & $24 \cdot 3$ & $25 \cdot 2$ \\
\hline$>60 \cdot 6$ & $21 \cdot 4$ & $26 \cdot 2$ & $22 \cdot 3$ & $26 \cdot 3$ & $22 \cdot 6$ & $26 \cdot 0$ \\
\hline Smoking status & & ** & & ** & & 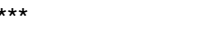 \\
\hline Non-smoker & $50 \cdot 5$ & $55 \cdot 3$ & $50 \cdot 9$ & $55 \cdot 6$ & $52 \cdot 0$ & $55 \cdot 0$ \\
\hline Former smoker & $33 \cdot 1$ & $33 \cdot 2$ & 33.7 & $32 \cdot 9$ & $32 \cdot 6$ & 33.4 \\
\hline Smoker & $16 \cdot 4$ & 11.5 & $15 \cdot 4$ & 11.5 & $15 \cdot 4$ & 11.6 \\
\hline Individual socio-economic factors & & & & & & \\
\hline Level of education & & ** & & ** & & 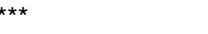 \\
\hline$<$ High school diploma & $10 \cdot 0$ & $10 \cdot 1$ & $10 \cdot 2$ & $10 \cdot 0$ & $10 \cdot 0$ & $10 \cdot 2$ \\
\hline Up to 2 years of university & 51.6 & 54.4 & $52 \cdot 6$ & $54 \cdot 2$ & 51.9 & $54 \cdot 4$ \\
\hline$>2$ years of university & 38.4 & 35.5 & $37 \cdot 2$ & $35 \cdot 8$ & $38 \cdot 1$ & $35 \cdot 4$ \\
\hline Occupation & & ** & & *** & & *** \\
\hline No & $20 \cdot 9$ & $32 \cdot 3$ & 23.0 & $32 \cdot 4$ & 23.5 & 31.9 \\
\hline Yes & 79.1 & $67 \cdot 7$ & $77 \cdot 0$ & $67 \cdot 6$ & 76.5 & 68.1 \\
\hline Women's income (n 50771; €/year) & & ** & & $\star * *$ & & *** \\
\hline$<16963$ & 11.5 & $9 \cdot 7$ & $11 \cdot 2$ & 9.8 & $11 \cdot 2$ & 9.7 \\
\hline $16963-17713$ & $27 \cdot 5$ & $28 \cdot 4$ & $27 \cdot 7$ & $28 \cdot 4$ & $27 \cdot 8$ & $28 \cdot 4$ \\
\hline $17713-24156$ & 18.4 & $20 \cdot 5$ & $19 \cdot 4$ & $20 \cdot 2$ & $18 \cdot 7$ & 20.5 \\
\hline$\geq 24156$ & $30 \cdot 2$ & 28.5 & $29 \cdot 2$ & 28.8 & $30 \cdot 0$ & $28 \cdot 4$ \\
\hline Number of children & & ** & & $\star * *$ & & 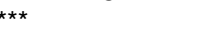 \\
\hline 0 & $10 \cdot 6$ & $11 \cdot 9$ & $10 \cdot 5$ & $12 \cdot 0$ & $11 \cdot 2$ & $11 \cdot 6$ \\
\hline 1 & $15 \cdot 4$ & $15 \cdot 2$ & $15 \cdot 2$ & $15 \cdot 3$ & $15 \cdot 9$ & $15 \cdot 0$ \\
\hline 2 & $45 \cdot 8$ & 43.5 & $45 \cdot 6$ & $43 \cdot 4$ & 44.9 & $43 \cdot 8$ \\
\hline$\geq 3$ & 28.2 & 29.4 & 28.7 & $29 \cdot 3$ & 28.0 & 29.6 \\
\hline Marital status & & * & & ז* & & \\
\hline Living without partner & $15 \cdot 3$ & $16 \cdot 1$ & $15 \cdot 1$ & $16 \cdot 2$ & $15 \cdot 7$ & $16 \cdot 0$ \\
\hline Living with a partner & $84 \cdot 7$ & 83.9 & 84.9 & 83.8 & $84 \cdot 3$ & $84 \cdot 0$ \\
\hline Contextual socio-economic factors & & & & & & \\
\hline Area of birth & & ** & & *** & & *** \\
\hline South & $23 \cdot 6$ & $30 \cdot 2$ & $25 \cdot 9$ & $29 \cdot 8$ & $24 \cdot 0$ & $30 \cdot 6$ \\
\hline West & $11 \cdot 2$ & $10 \cdot 8$ & $11 \cdot 2$ & $10 \cdot 8$ & $11 \cdot 2$ & $10 \cdot 8$ \\
\hline North & $18 \cdot 1$ & $12 \cdot 0$ & $16 \cdot 6$ & $12 \cdot 1$ & $17 \cdot 1$ & $12 \cdot 0$ \\
\hline East & $16 \cdot 7$ & $15 \cdot 5$ & $16 \cdot 3$ & $15 \cdot 6$ & $16 \cdot 8$ & $15 \cdot .4$ \\
\hline Centre & 8.3 & 9.4 & $8 \cdot 1$ & $9 \cdot 6$ & 9.0 & $9 \cdot 1$ \\
\hline Paris and suburbs & $15 \cdot 5$ & 14.4 & 14.9 & 14.6 & $15 \cdot 2$ & $14 \cdot 4$ \\
\hline Dom-Tom & 0.3 & 0.3 & 0.2 & 0.3 & 0.3 & 0.3 \\
\hline Abroad & $6 \cdot 3$ & 7.4 & $6 \cdot 8$ & $7 \cdot 2$ & $6 \cdot 4$ & 7.4 \\
\hline Area of residence & & ** & & $\star \star \star$ & & 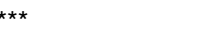 \\
\hline South & 28.7 & $37 \cdot 1$ & 31.6 & $36 \cdot \cdot 7$ & 29.2 & 37.5 \\
\hline West & 11.4 & 11.0 & 11.4 & $10 \cdot 9$ & 11.5 & $10 \cdot 9$ \\
\hline North & $16 \cdot 8$ & 11.1 & $15 \cdot 5$ & 11.2 & $15 \cdot 6$ & 11.3 \\
\hline East & $15 \cdot 6$ & $13 \cdot 8$ & $15 \cdot 1$ & $13 \cdot 8$ & $15 \cdot 8$ & 13.6 \\
\hline Centre & 7.4 & 8.6 & $7 \cdot 3$ & 8.7 & $8 \cdot 2$ & 8.3 \\
\hline Paris and suburbs & $20 \cdot 1$ & $18 \cdot 4$ & $19 \cdot 1$ & $18 \cdot 7$ & $19 \cdot 7$ & $18 \cdot 4$ \\
\hline $\begin{array}{l}\text { Size of the agglomeration of } \\
\text { residence }\end{array}$ & & & & & 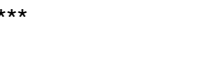 & \\
\hline Rural (<2000 inhabitants) & $17 \cdot 1$ & $16 \cdot 4$ & $17 \cdot 6$ & $16 \cdot 0$ & $16 \cdot 5$ & $16 \cdot 6$ \\
\hline $\begin{array}{l}\text { Quasi-rural (2000-9999 } \\
\text { inhabitants) }\end{array}$ & $26 \cdot 3$ & $26 \cdot 2$ & $26 \cdot 6$ & $26 \cdot 1$ & $26 \cdot 1$ & $26 \cdot 3$ \\
\hline
\end{tabular}




\begin{tabular}{|c|c|c|c|c|c|c|}
\hline \multirow[b]{2}{*}{ Percentage (\%) } & \multicolumn{2}{|c|}{ Fruit and vegetables } & \multicolumn{2}{|c|}{ Fruit } & \multicolumn{2}{|c|}{ Vegetables } \\
\hline & $\begin{array}{c}\text { Women } \\
\text { consuming less } \\
\text { than the ratiot } \\
(n 14972)\end{array}$ & $\begin{array}{l}\text { Women } \\
\text { consuming the } \\
\text { ratiot or more } \\
(n 43221)\end{array}$ & $\begin{array}{c}\text { Women } \\
\text { consuming less } \\
\text { than the ratiof } \\
(n 19007)\end{array}$ & $\begin{array}{l}\text { Women } \\
\text { consuming the } \\
\text { ratio } \neq \text { or more } \\
(n 39186)\end{array}$ & $\begin{array}{c}\text { Women } \\
\text { consuming less } \\
\text { than the ratio§ } \\
(n 17685)\end{array}$ & $\begin{array}{l}\text { Women } \\
\text { consuming the } \\
\text { ratio§ or more } \\
(n 40508)\end{array}$ \\
\hline $\begin{array}{l}\text { Quasi-urban (10 000-99999 } \\
\text { inhabitants) }\end{array}$ & $38 \cdot 7$ & $39 \cdot 1$ & $38 \cdot 3$ & $39 \cdot 4$ & $39 \cdot 3$ & 38.9 \\
\hline $\begin{array}{l}\text { Large urban areas }(\geq 100000 \\
\text { inhabitants) }\end{array}$ & $17 \cdot 9$ & $18 \cdot 3$ & $17 \cdot 5$ & $18 \cdot 5$ & $18 \cdot 1$ & $18 \cdot 2$ \\
\hline \multicolumn{7}{|l|}{ Deprivation index, FDep99 } \\
\hline$<-0.51$ (less deprived) & $32 \cdot 7$ & $33 \cdot 1$ & $32 \cdot 7$ & 33.1 & $32 \cdot 9$ & $33 \cdot 0$ \\
\hline $0.51-0.21$ & $32 \cdot 7$ & $33 \cdot 1$ & 33.0 & 33.0 & $32 \cdot 8$ & $33 \cdot 1$ \\
\hline$\geq 0.21$ & 34.6 & 33.8 & 34.3 & 33.9 & $34 \cdot 3$ & 33.9 \\
\hline $\begin{array}{l}\text { Size of the working agglomeration } \\
(n 33453)\end{array}$ & \multicolumn{2}{|c|}{$\star \star \star *$} & \multicolumn{2}{|c|}{ 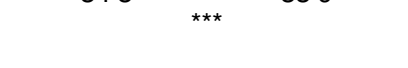 } & \multirow{2}{*}{\multicolumn{2}{|c|}{$* \star *$}} \\
\hline$\leq 10000$ inhabitants & $20 \cdot 0$ & $16 \cdot 4$ & $19 \cdot 6$ & $16 \cdot 3$ & & \\
\hline$>10000$ inhabitants & $45 \cdot 1$ & 38.4 & 43.5 & 38.5 & 43.9 & 38.5 \\
\hline
\end{tabular}

MET, metabolic equivalent of task.

Percentages were significantly different between both categories: ${ }^{\star} P<0.05,{ }^{\star \star} P<0.01,{ }^{* \star *} P<0.001$.

†The standard ratio used was $400 \mathrm{~g}$ fruit and vegetables $/ 1800 \mathrm{kcal}(400 \mathrm{~g} / 7530 \mathrm{~kJ})$, i.e. $0.22 \mathrm{~g} / \mathrm{kcal}(0.053 \mathrm{~g} / \mathrm{kJ})$.

$\ddagger$ The standard ratio used was $200 \mathrm{~g}$ fruit $/ 1800 \mathrm{kcal}(200 \mathrm{~g} / 7530 \mathrm{~kJ})$, i.e. $0.11 \mathrm{~g} / \mathrm{kcal}(0.027 \mathrm{~g} / \mathrm{kJ})$.

$\S$ The standard ratio used was $200 \mathrm{~g}$ vegetables $/ 1800 \mathrm{kcal}(200 \mathrm{~g} / 7530 \mathrm{~kJ})$, i.e. $0.11 \mathrm{~g} / \mathrm{kcal}(0.027 \mathrm{~g} / \mathrm{kJ})$.

occupation, respectively. Having an occupation was not associated with an increasing fruit consumption, but was associated with increasing grapefruit $(\mathrm{OR}=1 \cdot 14 ; 95 \% \mathrm{CI}$ $1.07,1.22)$, pear $(\mathrm{OR}=1 \cdot 14 ; 95 \% \mathrm{CI} 1.07,1.22)$ and grape (OR $=1.09 ; 95 \%$ CI 1.02, 1.17) consumption (Tables 2 and 3). The increase in fruit consumption was 96.2 and $69.0 \mathrm{~g} / \mathrm{d}$ on average for women with and with no occupation, respectively.

Intermediate incomes were modestly associated with a higher probability of increasing vegetable consumption $(\mathrm{OR}=1 \cdot 12 ; 95 \%$ CI $1.03,1.21$ for third $v$. first quartile for income; Table 2).

The association observed between having three or more children and the probability of increasing vegetable consumption was positive $(\mathrm{OR}=1.33$; $95 \%$ CI $1.24,1.42)$, including raw cucumber consumption $(\mathrm{OR}=1 \cdot 31$; $95 \% \mathrm{CI}$ 1.23, 1.40; Tables 2 and 3). The increase in vegetable consumption was 39.5 and $24.8 \mathrm{~g} / \mathrm{d}$ on average for women with three or more children and women with no child, respectively.

Living with a partner was associated with a modestly higher probability of increasing consumption of fruits $(\mathrm{OR}=1.07 ; 95 \% \mathrm{CI} 1.02,1.13)$ and raw vegetables (e.g. $\mathrm{OR}=1 \cdot 14 ; 95 \%$ CI $1.08,1.20$ for radish consumption; Tables 2 and 3).

\section{Contextual socio-economic factors associated with an increase in fruit and vegetable consumption}

When considering contextual socio-economic factors and the probability of increasing fruit and vegetable consumption, no association was observed except for a negative one concerning the size of the place of work $(\mathrm{OR}=0.90 ; 95 \%$ CI $0.85,0.96$ for cities with $>10000$ inhabitants $v$. smaller-sized cities; Tables 4 and 5). The increase in fruit and vegetable consumption was 139.8 and $161.6 \mathrm{~g} / \mathrm{d}$ on average for women working in bigger and smaller cities, respectively.

\section{Individual characteristics associated with an increase in fruit and vegetable consumption}

Being older was associated with a lower probability of increasing fruit and vegetable consumption over a 12-year period $(\mathrm{OR}=0.71 ; 95 \% \mathrm{CI} 0.67,0.77$ for fourth $v$. first quartile for age). The association was stronger for vegetable (OR $=0.63 ; 95 \%$ CI $0.59,0.67)$ than for fruit consumption (OR $=0.86 ; 95 \%$ CI $0.80,0.92)$ and was observed for each type of raw and cooked vegetables. Regarding fruit consumption, only grapefruit ( $\mathrm{OR}=0 \cdot 90$; $95 \%$ CI 0.84, 0.96) and pear (OR=0.88; $95 \%$ CI 0.82, 0.94) consumption showed the association (see online supplementary material, Supplemental Table 2). The increase in fruit and vegetable consumption was 162.2 and $70.8 \mathrm{~g} / \mathrm{d}$ on average for the youngest and oldest women, respectively.

Having a higher BMI was associated with an increase only in vegetable consumption $(\mathrm{OR}=1 \cdot 28$; $95 \%$ CI $1 \cdot 15$, 1.42 for obese $v$. normal BMI). All the vegetable subtypes studied showed the association except for courgette consumption $(\mathrm{OR}=1.08 ; 95 \%$ CI 0.97, 1.19; Supplemental Table 2).

Having a higher level of physical activity was positively associated with an increased fruit and vegetable consumption ( $\mathrm{OR}=1.21 ; 95 \% \mathrm{CI} 1.15,1.28$ for the fourth $v$. first quartile of physical activity). The same was observed for the most consumed subtypes of fruits and vegetables except for green beans (OR $=1.04 ; 95 \%$ CI 0.98, 1.09) and 
Table 2 Individual socio-economic factors associated with an increase in fruit and vegetable consumption between 1993 and 2005 using multivariable logistic regression models (E3N-EPIC cohort study, $n$ 58 193†)

\begin{tabular}{|c|c|c|c|c|c|c|c|c|c|c|c|c|}
\hline & \multicolumn{12}{|c|}{ Increase in the ratio of consumption to energy intake between 1993 and 2005} \\
\hline & \multicolumn{4}{|c|}{$\begin{array}{l}\text { Fruit and vegetables } \\
\qquad(n 37333)\end{array}$} & \multicolumn{4}{|c|}{$\begin{array}{c}\text { Fruit } \\
\text { (n 37304) }\end{array}$} & \multicolumn{4}{|c|}{$\begin{array}{l}\text { Vegetables } \\
\text { ( } n 32 \text { 993) }\end{array}$} \\
\hline & $n$ & $\%$ & OR $\ddagger$ & $95 \% \mathrm{Cl}$ & $n$ & $\%$ & OR & $95 \% \mathrm{Cl}$ & $n$ & $\%$ & OR $\ddagger$ & $95 \% \mathrm{Cl}$ \\
\hline \multicolumn{13}{|l|}{ Level of education } \\
\hline$<$ High school diploma & 3537 & $60 \cdot 1$ & \multicolumn{2}{|c|}{ Ref. } & 3647 & $62 \cdot 0$ & \multicolumn{2}{|c|}{ Ref. } & 3012 & $51 \cdot 2$ & \multicolumn{2}{|c|}{ Ref. } \\
\hline Up to 2 years of university & 20025 & $64 \cdot 1$ & 1.14 & $1.05,1.23$ & 20014 & $64 \cdot 1$ & 1.07 & $0.99,1.15$ & 17702 & $56 \cdot 7$ & 1.18 & $1.09,1.27$ \\
\hline$>2$ years of university & 13771 & $65 \cdot 3$ & 1.16 & $1.07,1.26$ & 13643 & 64.7 & 1.09 & $1.00,1 \cdot 18$ & 12279 & $58 \cdot 2$ & $1 \cdot 17$ & $1.08,1.27$ \\
\hline \multicolumn{13}{|l|}{ Occupation } \\
\hline No & 9750 & $57 \cdot 1$ & \multicolumn{2}{|c|}{ Ref. } & 10161 & 59.5 & \multicolumn{2}{|c|}{ Ref. } & 8431 & 49.4 & \multicolumn{2}{|c|}{ Ref. } \\
\hline Yes & 27583 & $67 \cdot 1$ & 1.21 & $1.12,1.30$ & 27143 & $65 \cdot 6$ & 1.03 & $0.96,1 \cdot 11$ & 24562 & $59 \cdot 7$ & 1.20 & $1 \cdot 12,1.28$ \\
\hline \multicolumn{13}{|l|}{ Women's income ( $€ /$ year) } \\
\hline$<16963$ & 3828 & 64.5 & \multicolumn{2}{|c|}{ Ref. } & 3855 & 65.0 & \multicolumn{2}{|c|}{ Ref. } & 3260 & $55 \cdot 0$ & \multicolumn{2}{|c|}{ Ref. } \\
\hline $16963-17713$ & 10641 & 64.9 & 1.04 & $0.96,1.13$ & 10532 & $64 \cdot 2$ & 1.00 & $0.93,1.08$ & 9401 & $57 \cdot 3$ & 1.11 & $1.02,1.20$ \\
\hline $17713-24156$ & 7385 & $63 \cdot 6$ & 1.02 & $0.94,1.11$ & 7459 & $64 \cdot 2$ & 1.02 & $0.94,1.11$ & 6527 & $56 \cdot 2$ & 1.12 & $1.03,1.21$ \\
\hline$\geq 24156$ & 10920 & 64.9 & 0.97 & $0.89,1.05$ & 10841 & 64.5 & 0.97 & $0.89,1.05$ & 9746 & 57.9 & 1.08 & $0.99,1.17$ \\
\hline \multicolumn{13}{|l|}{ Number of children } \\
\hline 0 & 4043 & $60 \cdot 4$ & \multicolumn{2}{|c|}{ Ref. } & 4144 & 61.9 & \multicolumn{2}{|c|}{ Ref. } & 3544 & $52 \cdot 9$ & \multicolumn{2}{|c|}{ Ref. } \\
\hline 1 & 5677 & 63.9 & 1.11 & $1.03,1.20$ & 5654 & 63.7 & 1.02 & $0.95,1.10$ & 5044 & $56 \cdot 8$ & 1.13 & $1.05,1.22$ \\
\hline 2 & 16770 & $65 \cdot 3$ & 1.19 & $1.11,1.27$ & 16769 & $65 \cdot 3$ & 1.08 & $1.01,1.15$ & 14739 & 57.4 & 1.19 & $1.11,1.27$ \\
\hline$\geq 3$ & 10843 & $64 \cdot 0$ & 1.24 & $1.16,1.33$ & 10737 & 63.4 & 1.05 & $0.98,1.12$ & 9666 & $57 \cdot 0$ & 1.33 & $1.24,1.42$ \\
\hline \multicolumn{13}{|l|}{ Marital status } \\
\hline Living without partner & 5690 & 61.5 & \multicolumn{2}{|c|}{ Ref. } & 5703 & $61 \cdot 7$ & \multicolumn{2}{|c|}{ Ref. } & 5063 & 54.8 & \multicolumn{2}{|c|}{ Ref. } \\
\hline Living with a partner & 31643 & 64.6 & 1.05 & $1.00,1 \cdot 11$ & 31601 & $64 \cdot 6$ & 1.07 & $1.02,1.13$ & 27930 & $57 \cdot 1$ & 1.03 & $0.98,1.09$ \\
\hline
\end{tabular}

Models are multi-adjusted and further adjusted for individual characteristics, contextual socio-economic factors, energy intake evolution between 1993 and 2005 and baseline consumption of the food group considered. Significant results are indicated in bold font.

†Information on income was available for 50771 women.

$\ddagger$ An OR above 1 corresponds to a higher probability for women to increase their ratio of consumption to energy intake between 1993 and 2005 . 


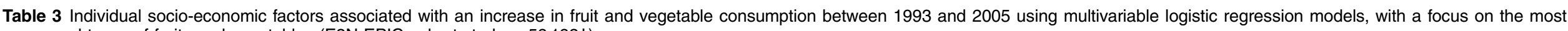
consumed types of fruits and vegetables (E3N-EPIC cohort study, $n 58193 \dagger$ )

\begin{tabular}{|c|c|c|c|c|c|c|c|c|c|c|c|c|c|c|c|c|c|c|c|c|}
\hline & \multicolumn{20}{|c|}{ crease in the ratio of consumption to energy intake between 1993 and 2005} \\
\hline & \multicolumn{10}{|c|}{ Most consumed fruits (mean (so) intake in 1993 in g) } & \multicolumn{10}{|c|}{ Most consumed cooked vegetables (mean (sD) intake in 1993 in g) } \\
\hline & \multicolumn{2}{|c|}{$\begin{array}{c}\text { Grapefruit } \\
(19 \cdot 8(18.0)) \\
(n 28024)\end{array}$} & \multicolumn{2}{|c|}{$\begin{array}{c}\text { Apple } \\
(26.8(20.5)) \\
(n 34383)\end{array}$} & \multicolumn{2}{|c|}{$\begin{array}{c}\text { Pear } \\
(18.2(14.8)) \\
(n 32766)\end{array}$} & \multicolumn{2}{|c|}{$\begin{array}{l}\text { Peach, nectarine } \\
(28 \cdot 0(20.0)) \\
(n 33088)\end{array}$} & \multicolumn{2}{|c|}{$\begin{array}{c}\text { Grapes } \\
(18 \cdot 1(14 \cdot 5)) \\
(n 31391)\end{array}$} & \multicolumn{2}{|c|}{$\begin{array}{c}\text { Green beans } \\
(19.7(13 \cdot 1)) \\
(n 26795)\end{array}$} & \multicolumn{2}{|c|}{$\begin{array}{c}\text { Courgette } \\
(15.2(11.4)) \\
(n 30564)\end{array}$} & \multicolumn{2}{|c|}{$\begin{array}{c}\text { Carrot } \\
(14.7(10.7)) \\
(n 28294)\end{array}$} & \multicolumn{2}{|c|}{$\begin{array}{c}\text { Tomato } \\
(13.3(9.8)) \\
(n 26388)\end{array}$} & \multicolumn{2}{|c|}{$\begin{array}{c}\text { Endive } \\
(13 \cdot 0(10.4)) \\
(n 28702)\end{array}$} \\
\hline & OR $\ddagger$ & $95 \% \mathrm{Cl}$ & OR $\ddagger$ & $95 \% \mathrm{Cl}$ & OR $\ddagger$ & $95 \% \mathrm{Cl}$ & OR $\ddagger$ & $95 \% \mathrm{Cl}$ & OR $\ddagger$ & $95 \% \mathrm{Cl}$ & OR $\ddagger$ & $95 \% \mathrm{Cl}$ & OR $\ddagger$ & $95 \% \mathrm{Cl}$ & OR $\ddagger$ & $95 \% \mathrm{Cl}$ & OR $\ddagger$ & $95 \% \mathrm{Cl}$ & OR $\ddagger$ & $95 \% \mathrm{Cl}$ \\
\hline \multicolumn{21}{|c|}{ Level of education (Ref. $=<$ high school diploma) } \\
\hline $\begin{array}{l}\text { Up to } 2 \text { years of university } \\
>2 \text { years of university }\end{array}$ & $\begin{array}{l}1 \cdot 10 \\
1 \cdot 16\end{array}$ & $\begin{array}{l}1 \cdot 02,1 \cdot 18 \\
1 \cdot 08,1 \cdot 25\end{array}$ & $\begin{array}{l}1.06 \\
1.08\end{array}$ & $\begin{array}{l}0.99,1 \cdot 14 \\
1 \cdot 00,1 \cdot 17\end{array}$ & $\begin{array}{l}1.09 \\
1.09\end{array}$ & $\begin{array}{l}1 \cdot 01,1 \cdot 17 \\
1 \cdot 01,1 \cdot 18\end{array}$ & $\begin{array}{l}1 \cdot 11 \\
1.09\end{array}$ & $\begin{array}{l}1 \cdot 03,1 \cdot 20 \\
1 \cdot 01,1 \cdot 18\end{array}$ & $\begin{array}{l}1.03 \\
1.02\end{array}$ & $\begin{array}{l}0.96,1 \cdot 11 \\
0.95,1.11\end{array}$ & $\begin{array}{l}1 \cdot 10 \\
1.09\end{array}$ & $\begin{array}{l}1 \cdot 02,1 \cdot 19 \\
1 \cdot 01,1 \cdot 19\end{array}$ & $\begin{array}{l}1.20 \\
1.27\end{array}$ & $\begin{array}{l}1 \cdot 11,1 \cdot 29 \\
1 \cdot 17,1 \cdot 37\end{array}$ & $\begin{array}{l}1 \cdot 15 \\
1 \cdot 15\end{array}$ & $\begin{array}{l}1 \cdot 06,1 \cdot 24 \\
1 \cdot 06,1 \cdot 25\end{array}$ & $\begin{array}{l}1 \cdot 12 \\
1 \cdot 14\end{array}$ & $\begin{array}{l}1 \cdot 04,1 \cdot 21 \\
1 \cdot 05,1 \cdot 23\end{array}$ & $\begin{array}{l}1 \cdot 13 \\
1 \cdot 10\end{array}$ & $\begin{array}{l}1 \cdot 05,1 \cdot 22 \\
1 \cdot 02,1 \cdot 19\end{array}$ \\
\hline & & & & & & & & \\
\hline \multirow{2}{*}{\multicolumn{21}{|c|}{ Women income ( $(\epsilon /$ year) $($ Ref. $=<16963)$}} \\
\hline & & & & & & & & & & & & & & & & & & & & \\
\hline $17713-24156$ & 1.09 & 1.01 & 1. & 0.98 & & & & & & & 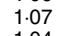 & & & & 1 & & 1.13 & & 0.99 & $0.92,1$ \\
\hline \multicolumn{21}{|l|}{$\begin{array}{l}\geq 24156 \\
\text { Number of children (Ref. }=0)\end{array}$} \\
\hline $\begin{array}{l}\text { Number of children (Ret. }=0 \text { ) } \\
1\end{array}$ & 1.0 & 0.5 & 1 & 0 & 1 & 0 & 1 & 1. & 1. & 1.0 & 1. & 0.9 & $1 \cdot 1$ & 1.05 & 1.0 & 0.9 & 1.08 & 1.01 & 0.99 & $0.93,1.07$ \\
\hline 2 & $1 \cdot 1$ & & & & & & & & & & 1. & & 1. & 1. & 1 & 0 & 1. & & 0.98 & $0.92,1$ \\
\hline \multirow{2}{*}{\multicolumn{21}{|c|}{$\begin{array}{l}\geq 3 \\
\text { Marital status (Ref. = living without partner) } \\
1 \cdot 05,1 \cdot 19\end{array}$}} \\
\hline & & & 1.04 & $0.98,1.09$ & $1 \cdot 12$ & $1 \cdot 06,1 \cdot 17$ & 1.09 & $1 \cdot 03,1 \cdot 15$ & 1.09 & $1 \cdot 03,1 \cdot 14$ & 0.97 & $0.92,1.03$ & 0.92 & $0.87,0.97$ & 1.02 & $0.97,1.08$ & 1.03 & $0.98,1.09$ & 1.02 & $0.97,1.08$ \\
\hline
\end{tabular}

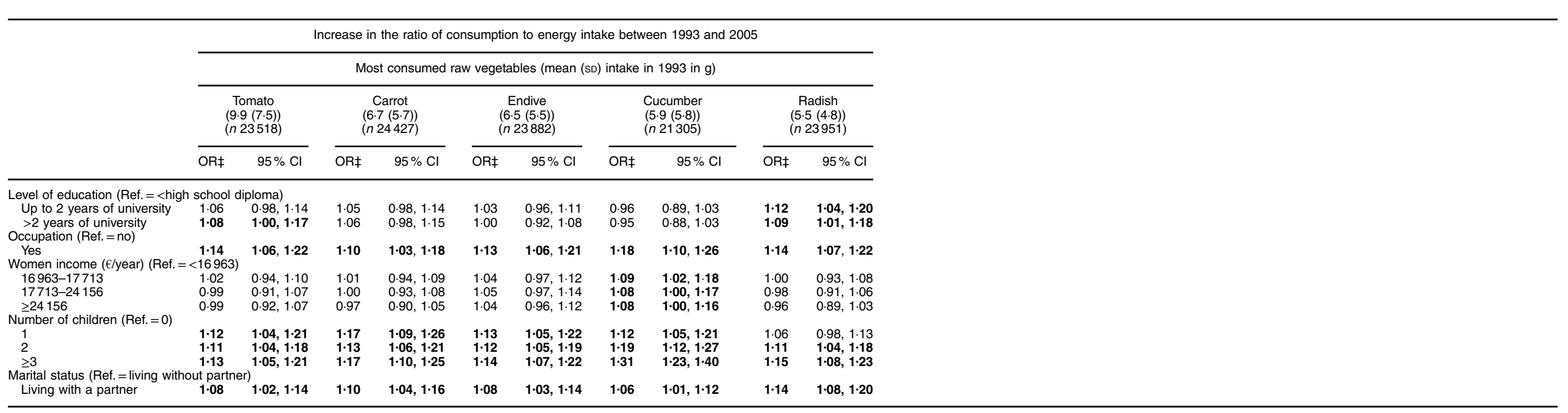

Ref refer

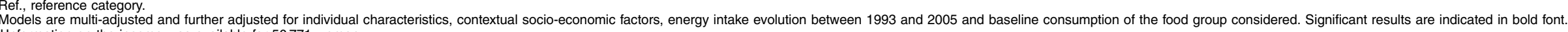
fAn OR above 1 corresponds to a higher probability for women to increase their ratio of consumption to energy intake between 1993 and 2005. 
endive $(\mathrm{OR}=1.04 ; 95 \%$ CI $0.99, \quad 1 \cdot 10 ;$ Supplemental Table 2).

Current smoking was associated with a lower probability of increasing fruit $(\mathrm{OR}=0.81 ; 95 \% \mathrm{CI} 0 \cdot 76,0 \cdot 85)$ and carrot and tomato consumptions (OR $=0.85 ; 95 \% \mathrm{CI}$ $0.81,0.90$ and $\mathrm{OR}=0.93 ; 95 \% \mathrm{CI} 0.88,0.99$, respectively). It was also associated with a higher probability of increasing raw endive and cucumber consumptions $(\mathrm{OR}=1 \cdot 06 ; 95 \% \mathrm{CI} 1 \cdot 00,1 \cdot 12$ and $\mathrm{OR}=1 \cdot 12 ; 95 \% \mathrm{CI} 1.06$, $1 \cdot 19$, respectively). Former smokers tended to decrease or maintain their apple and pear consumptions $(\mathrm{OR}=0.95$; $95 \%$ CI $0.91,0.98$ and $\mathrm{OR}=0.94 ; 95 \%$ CI $0.90,0.97$, respectively) and to increase their raw endive, cucumber and radish consumptions (OR $=1.05 ; 95 \%$ CI 1.01, 1.09, $\mathrm{OR}=1 \cdot 11 ; 95 \%$ CI $1.07,1.15$ and $\mathrm{OR}=1.05 ; 95 \%$ CI 1.01 , 1.09, respectively; Supplemental Table 2).

\section{Discussion}

In the large E3N-EPIC cohort, our study investigated the socio-economic factors associated with an increase in fruit and vegetable consumption over a 12 -year period. To our knowledge, this is the first prospective study to simultaneously consider the impact of individual and contextual socio-economic factors on changes in fruit and vegetable consumption. In agreement with previously published cross-sectional studies ${ }^{(25,53)}$, our results show that long-term changes in fruit and vegetable consumption are socio-economically patterned. However, the socioeconomic factors associated with an increase in fruit consumption were not the same as those associated with an increase in vegetable consumption. The associations also differed according to fruit and vegetable subtypes.

It has previously been suggested that environmental determinants of fruit consumption and vegetable consumption could be different ${ }^{(18)}$. For example, the presence of fruits in the fruit bowl on the table at home may elicit fruit consumption and culture-specific eating patterns may determine the amount and the type of vegetables eaten during meals.

In our study, associations between socio-economic status and changes in fruit and vegetable consumption over a 12 -year period were observed for individual socioeconomic factors but not for contextual factors. Some studies have shown that contextual factors such as neighbourhood socio-economic status are associated with fruit and vegetable consumption ${ }^{(54-57)}$, wherein individuals living in neighbourhoods with higher socioeconomic status had higher intakes of fruits and vegetables; however, other studies were less conclusive in that respect ${ }^{(58,59)}$. Defining the contextual aspect of the socio-economic environment and selecting relevant variables to study have been reported to be complex ${ }^{(48,60,61)}$. Therefore, there is still room to understand the complex interactions between individual and contextual factors to act upon. In our study, all the considered contextual socioeconomic factors were found to be unrelated to fruit and vegetable consumption changes over time. It would be interesting to replicate our study design with other contextual socio-economic factors than the ones available in the E3N study.

Higher education and physical activity levels were associated with an increase in fruit and vegetable consumption. Level of education and physical activity have previously been shown to be positively associated with fruit and vegetable consumption in cross-sectional studies ${ }^{(41,49,62)}$. Educational level may be associated with an increase in fruit and vegetable consumption through several pathways such as dietary knowledge and ability to interpret health education messages ${ }^{(23,63)}$. Regarding physical activity, our results suggest a global awareness about health and a combination of positive healthy behaviours. Having an occupation and an increased number of children were associated with a higher probability of increasing vegetable consumption. Positive associations between occupation level and consumption of both fruits and vegetables have been previously demonstrated in cross-sectional studies as well ${ }^{(17,41)}$, whereas having children provided mixed results ${ }^{(18)}$. Our finding that living with a partner was associated with a higher probability of increasing fruit consumption is in line with previous data ${ }^{(18)}$. Living with a partner may affect a person's fruit and vegetable intake via the partner's eating patterns, social support, sociocultural norms and home availability of fruits and vegetables ${ }^{(18)}$. In France fruits and vegetables are sold by the kilogram, while other countries such as the UK tend to sell fruits and vegetables in smaller prepacked portions. Therefore, French individuals who live on their own may be less prone to buy fruits and vegetables as well as to do home cooking of vegetables, especially while they are ageing. Also, social networks and supportive social norms have been suggested to be strongly associated with changes in fruit and vegetable consumption $^{(64)}$. This may partly explain the positive associations we observed between having an occupation, being in a couple, having several children and the increase in fruit and vegetable consumption over time.

When considering the descriptive characteristics of the population in 1993, in comparison with women having fruit and vegetable consumption below the standard ratio, women with consumption greater than or equal to the standard ratio had similar level of education, number of children and marital status, and were less frequently with a professional activity. These results could suggest that the National Nutrition and Health Program ${ }^{(9,18)}$, which started to promote the consumption of five servings of fruits and vegetables daily in 2001 with the help of radio and television announcements, was likely to have a greater impact on women living with a partner, with high education level, with an increased number of children and without professional activity. Women with high physical activity levels were already more likely to follow 


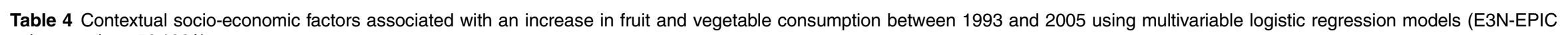
cohort study, $n 58193 \dagger)$

\begin{tabular}{|c|c|c|c|c|c|c|c|c|c|c|c|c|}
\hline & \multicolumn{4}{|c|}{$\begin{array}{l}\text { Fruit and vegetables } \\
\qquad(n 37333)\end{array}$} & \multicolumn{4}{|c|}{$\begin{array}{c}\text { Fruit } \\
(n 37304)\end{array}$} & \multicolumn{4}{|c|}{$\begin{array}{l}\text { Vegetables } \\
\text { (n 32 993) }\end{array}$} \\
\hline & $n$ & $\%$ & OR $\ddagger$ & $95 \% \mathrm{Cl}$ & $n$ & $\%$ & OR $\ddagger$ & $95 \% \mathrm{Cl}$ & $n$ & $\%$ & OR $\ddagger$ & $95 \% \mathrm{Cl}$ \\
\hline \multicolumn{13}{|l|}{ Area of birth } \\
\hline South & 10491 & 63.2 & \multicolumn{2}{|c|}{ Ref. } & 10454 & $62 \cdot 9$ & \multicolumn{2}{|c|}{ Ref. } & 9409 & $56 \cdot 6$ & \multicolumn{2}{|c|}{ Ref. } \\
\hline West & 4081 & $64 \cdot 2$ & 0.93 & $0.86,1.01$ & 4059 & $63 \cdot 9$ & 0.96 & $0.89,1.04$ & 3596 & $56 \cdot 6$ & 0.87 & $0.81,0.95$ \\
\hline North & 5294 & $67 \cdot 0$ & 0.96 & $0.89,1.04$ & 5230 & $66 \cdot 2$ & 0.96 & $0.89,1.04$ & 4589 & $58 \cdot 1$ & 0.88 & $0.82,0.95$ \\
\hline East & 5930 & 64.4 & 0.98 & $0.91,1.06$ & 5961 & 64.7 & 1.04 & $0.96,1.12$ & 5213 & $56 \cdot 6$ & 0.89 & $0.83,0.96$ \\
\hline Centre & 3448 & $65 \cdot 2$ & 1.04 & $0.95,1.13$ & 3456 & $65 \cdot 3$ & 1.07 & $0.99,1.16$ & 3015 & $57 \cdot 0$ & 0.96 & $0.88,1.04$ \\
\hline Paris and suburbs & 5406 & 63.3 & 0.93 & $0.86,0.99$ & 5464 & 64.0 & 1.00 & $0.93,1.07$ & 4772 & $55 \cdot 9$ & 0.88 & $0.82,0.94$ \\
\hline Dom-Tom & 99 & 61.9 & 0.89 & $0.63,1.28$ & 99 & 61.9 & 0.98 & $0.69,1.39$ & 84 & $52 \cdot 5$ & 0.76 & $0.53,1.07$ \\
\hline Abroad & 2584 & 62.5 & 0.96 & $0.89,1.04$ & 2581 & $62 \cdot 4$ & 0.95 & $0.88,1.03$ & 2315 & $56 \cdot 0$ & 0.99 & $0.92,1.07$ \\
\hline \multicolumn{13}{|l|}{ Area of residence } \\
\hline South & 12786 & $62 \cdot 7$ & \multicolumn{2}{|c|}{ Ref. } & 12783 & $62 \cdot 7$ & \multicolumn{2}{|c|}{ Ref. } & 11393 & $55 \cdot 9$ & \multicolumn{2}{|c|}{ Ref. } \\
\hline West & 4209 & $65 \cdot 2$ & 1.05 & $0.97,1.14$ & 4150 & $64 \cdot 3$ & 1.03 & $0.96,1.12$ & 3753 & 58.1 & 1.07 & $0.99,1.16$ \\
\hline North & 4925 & $67 \cdot 3$ & 0.99 & $0.91,1.08$ & 4899 & $66 \cdot 9$ & 1.05 & $0.97,1.13$ & 4245 & $58 \cdot 0$ & 0.93 & $0.86,1.01$ \\
\hline East & 5367 & 64.7 & 0.91 & $0.84,0.98$ & 5372 & 64.8 & 0.96 & $0.89,1.03$ & 4759 & 57.4 & 0.92 & $0.85,0.99$ \\
\hline Centre & 3098 & 64.5 & 1.03 & $0.94,1.12$ & 3132 & $65 \cdot 2$ & $1 \cdot 11$ & $1 \cdot 02,1 \cdot 20$ & 2685 & 55.9 & 0.93 & $0.86,1.01$ \\
\hline Paris and suburbs & 6948 & 63.5 & 0.95 & $0.89,1.01$ & 6968 & 63.6 & 0.96 & $0.89,1.02$ & 6158 & $56 \cdot 2$ & 0.95 & $0.89,1.01$ \\
\hline \multicolumn{13}{|l|}{ Size of the agglomeration of residence } \\
\hline Rural ( $<2000$ inhabitants) & 6287 & $65 \cdot 3$ & \multicolumn{2}{|c|}{ Ref. } & 6260 & $65 \cdot 0$ & \multicolumn{2}{|c|}{ Ref. } & 5526 & 57.4 & \multicolumn{2}{|c|}{ Ref. } \\
\hline Quasi-rural (2000-9999 inhabitants) & 9902 & 64.8 & 0.98 & $0.92,1.04$ & 9880 & 64.6 & 1.01 & $0.95,1.07$ & 8785 & 57.5 & 1.00 & $0.95,1.06$ \\
\hline Quasi-urban (10 000-99 999 inhabitants) & 14522 & $64 \cdot 0$ & 1.00 & $0.94,1.06$ & 14528 & 64.0 & 1.03 & $0.97,1.09$ & 12755 & $56 \cdot 2$ & 0.99 & $0.94,1.05$ \\
\hline Large urban areas ( $\geq 100000$ inhabitants) & 6622 & 62.6 & 0.97 & $0.91,1.04$ & 6636 & $62 \cdot 8$ & 1.01 & $0.94,1.08$ & 5927 & $56 \cdot 0$ & 1.00 & $0.94,1.07$ \\
\hline \multicolumn{13}{|l|}{ Deprivation index, FDep99 } \\
\hline$<-0.51$ (less deprived) & 12251 & 63.8 & \multicolumn{2}{|c|}{ Ref. } & 12324 & $64 \cdot 2$ & \multicolumn{2}{|c|}{ Ref. } & 10869 & $56 \cdot 6$ & \multicolumn{2}{|c|}{ Ref. } \\
\hline $0.51-0.21$ & 12340 & $64 \cdot 3$ & 1.01 & $0.96,1.06$ & 12279 & 63.9 & 0.97 & $0.92,1.02$ & 10979 & $57 \cdot 2$ & 1.01 & $0.96,1.06$ \\
\hline$\geq 0.21$ & 12742 & $64 \cdot 4$ & 0.99 & $0.94,1.05$ & 12701 & $64 \cdot 2$ & 0.96 & $0.91,1.01$ & 11145 & $56 \cdot 3$ & 0.96 & $0.92,1.01$ \\
\hline \multicolumn{13}{|l|}{ Size of the working agglomeration } \\
\hline$\leq 10000$ inhabitants & 7016 & 69.5 & \multicolumn{2}{|c|}{ Ref. } & 6862 & 68.0 & \multicolumn{2}{|c|}{ Ref. } & 6291 & $62 \cdot 3$ & \multicolumn{2}{|c|}{ Ref. } \\
\hline$>10000$ inhabitants & 15497 & $66 \cdot 3$ & 0.90 & $0.85,0.96$ & 15326 & $65 \cdot 6$ & 0.92 & $0.87,0.98$ & 13776 & $59 \cdot 0$ & 0.89 & $0.84,0.95$ \\
\hline
\end{tabular}

Ref., reference category. Models are multi-adjusted and further adjusted for individual characteristics, contextual socio-economic factors, energy intake evolution between 1993 and 2005 and baseline consumption of the food group considered. in bold font.

łAn OR above 1 corresponds to a higher probability for women to increase their ratio of consumption to energy intake between 1993 and 2005. 
Table 5 Contextual socio-economic factors associated with an increase in fruit and vegetable consumption between 1993 and 2005 using multivariable logistic regression models, with a focus on the most consumed types of fruits and vegetables (E3N-EPIC cohort study, $n 58193 \dagger$ )

\begin{tabular}{|c|c|c|c|c|c|c|c|c|c|c|c|c|c|c|c|c|c|c|c|c|}
\hline & \multicolumn{20}{|c|}{ Increase in the ratio of consumption to energy intake between 1993 and 2005} \\
\hline & \multicolumn{10}{|c|}{ Most consumed fruits (mean (SD) intake in 1993 in g) } & \multicolumn{10}{|c|}{ Most consumed cooked vegetables (mean (sD) intake in 1993 in g) } \\
\hline & \multicolumn{2}{|c|}{$\begin{array}{c}\text { Grapefruit } \\
(19.8(18.0)) \\
(n 28024)\end{array}$} & \multicolumn{2}{|c|}{$\begin{array}{c}\text { Apple } \\
(26.8(20.5)) \\
(n 34383)\end{array}$} & \multicolumn{2}{|c|}{$\begin{array}{c}\text { Pear } \\
(18.2(14.8)) \\
(n 32766) \\
\end{array}$} & \multicolumn{2}{|c|}{$\begin{array}{c}\text { Peach, nectarine } \\
(28.0(20.0)) \\
(n 33088) \\
\end{array}$} & \multicolumn{2}{|c|}{$\begin{array}{c}\text { Grapes } \\
\left(\begin{array}{c}\text { G.1 (14.5)) } \\
(n 31391)\end{array}\right. \\
\end{array}$} & \multicolumn{2}{|c|}{$\begin{array}{c}\text { Green beans } \\
\left(\begin{array}{c}(19.7(13.1)) \\
(n 26795)\end{array}\right. \\
\end{array}$} & \multicolumn{2}{|c|}{$\begin{array}{c}\text { Courgette } \\
(15.2(11.4)) \\
(n 30564)\end{array}$} & \multicolumn{2}{|c|}{$\begin{array}{c}\text { Carrot } \\
(14.7(10.7)) \\
(n 28294)\end{array}$} & \multicolumn{2}{|c|}{$\begin{array}{c}\text { Tomato } \\
(13.3(9.8)) \\
(n 26388) \\
\end{array}$} & \multicolumn{2}{|c|}{$\begin{array}{c}\text { Endive } \\
(13.0(10.4)) \\
(n 28702) \\
\end{array}$} \\
\hline & OR $\ddagger$ & $95 \% \mathrm{Cl}$ & OR $\ddagger$ & $95 \% \mathrm{Cl}$ & OR $\ddagger$ & $95 \% \mathrm{Cl}$ & OR $\ddagger$ & $95 \% \mathrm{Cl}$ & OR $\ddagger$ & $95 \% \mathrm{Cl}$ & OR $\ddagger$ & $95 \% \mathrm{Cl}$ & OR $\ddagger$ & $95 \% \mathrm{Cl}$ & OR $\ddagger$ & $95 \% \mathrm{Cl}$ & OR & $95 \% \mathrm{Cl}$ & OR & $95 \% \mathrm{Cl}$ \\
\hline \multicolumn{21}{|l|}{ Area of birth (Ref. $=$ South) } \\
\hline West & 1.02 & $0.94,1 \cdot 10$ & 1.03 & 0.95 & 1.01 & $0.93,1.09$ & 0.97 & $0.90,1.05$ & 0.92 & $0.86,1.00$ & 0.96 & $0.88,1.04$ & 0.81 & $0.75,0.88$ & 0.93 & $0.86,1.01$ & 0.87 & $0.81,0.94$ & 0.79 & $0.73,0.85$ \\
\hline & 1.05 & & 0.95 & & 1.05 & & & & 0.94 & & 0.92 & & 0.85 & & 0.96 & & & & & \\
\hline & 1.06 & 0.99 & 1.02 & 0.95 & 0.98 & 0.91, & 0.98 & & 0.98 & 0.9 & 0.97 & 0.9 & 0.91 & & 1.00 & & 0.85 & & 0.87 & \\
\hline Centr & 1.05 & $0.97,1.13$ & 1.02 & 0.94 & 1.05 & $0.97,1.13$ & 1.05 & 0.97 & 0.96 & 0.89 & 0.96 & $0.88,1.04$ & 0.94 & & 0.98 & & 0.95 & & 0.94 & \\
\hline $\mathrm{P}$ & 1.00 & $0.94,1.06$ & 0.91 & $0.85, c$ & 1.00 & $0.94,1.06$ & 0.97 & $0.91,1.04$ & 0.95 & $0.89,1.01$ & 0.94 & $0.88,1.00$ & 0.88 & $0.83,0.94$ & 0.92 & 0.86 & 0.87 & & 0.87 & \\
\hline & 1.12 & $0.81,1.54$ & 0.73 & $0.52,1.02$ & 0.73 & $0.53,1.02$ & 0.67 & $0.48,0.93$ & 0.90 & $0.65,1.25$ & 0.86 & $0.61,1.22$ & 0.90 & $0.64,1.27$ & 0.80 & $0.57,1.12$ & 0.88 & $0.63,1.24$ & 0.78 & $0.56,1.09$ \\
\hline A & 0.99 & $0.92,1.07$ & 0.86 & $0.80,0.93$ & 0.94 & $0.87,1.01$ & 0.89 & 0.83, & 0.98 & $0.91,1.06$ & 0.90 & $0.84,0.98$ & 1.04 & $0.96,1.12$ & 0.94 & $0.87,1.01$ & 0.90 & $0.83,0.97$ & 0.83 & $0.77,0.90$ \\
\hline \multicolumn{21}{|c|}{ 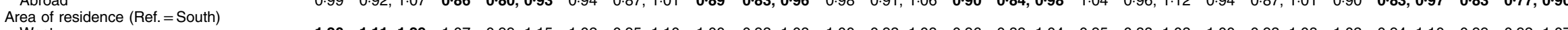 } \\
\hline West & $1 \cdot 20$ & $1 \cdot 11$ & 1.07 & & 1.02 & & & & 1.00 & & 0.96 & & 5 & & & & & & & \\
\hline & $1 \cdot 10$ & 1.02 & 1.06 & & & & 1.01 & & 1.00 & & & & 0.89 & & 1.03 & & 0.90 & & 1.05 & \\
\hline & 1.07 & 1.00 & 1.03 & & 0.94 & & 0.95 & & 0.99 & & 0.91 & & 0.89 & & 1.01 & & 0.87 & & 0.92 & \\
\hline & $1 \cdot 14$ & 1.05, & $1 \cdot 15$ & & 1.08 & & 1.00 & & 0.99 & & & & 0.79 & & & & & & 96 & \\
\hline 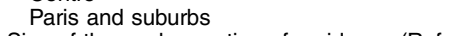 & 1.04 & $0.98,1.10$ & 0.99 & 0.93 & 0.98 & 0.9 & 0.97 & 3 & 0.94 & 0.8 & 0.99 & 0.9 & 0.93 & 0.8 & 0.99 & & 0.90 & .96 & 0.99 & 1.05 \\
\hline \multicolumn{21}{|c|}{ Size of the agglomeration of residence (Ref. $=$ rural $(<2000$ inhabitants $))$} \\
\hline Quasi-rural (2000-9999 inhabitants) & 1.04 & $0.98,1.09$ & 0.96 & $0.91,1.02$ & 1.04 & $0.98,1.09$ & 1.05 & & 1.01 & & 1.00 & & 1.01 & & 1.06 & & 0.99 & & 1.08 & \\
\hline & 1.06 & & 0.99 & & 1.06 & & & & 1.04 & & 1.01 & & 1.02 & & 1.04 & & 0.98 & & 1.06 & \\
\hline \multirow{2}{*}{\multicolumn{21}{|c|}{ Deprivation index, FDep99 (Ref. $=<-0.51$ (less deprived)) }} \\
\hline & & & & & & & & & & & & & & & & & & & & \\
\hline & 0.96 & & 0.96 & & 0.96 & $0.91,1.00$ & 0.96 & $0.92,1.01$ & 0.98 & $0.93,1.02$ & 1.01 & $0.96,1.06$ & 1.01 & $0.96,1.06$ & 1.01 & $0.96,1.06$ & 0.99 & $0.94,1.04$ & 0.98 & 0.94 \\
\hline$\geq 0.21$ & 0.91 & $0.87,0.96$ & 0.97 & $0.92,1.02$ & 0.99 & $0.94,1.04$ & 0.97 & $0.92,1.02$ & 0.98 & $0.93,1.03$ & 0.98 & $0.93,1.03$ & 0.98 & $0.93,1.03$ & 0.99 & $0.94,1.04$ & 1.00 & $0.95,1.05$ & 0.97 & $0.92,1.02$ \\
\hline $\begin{array}{l}\text { Size of the working agglomeration (Ref. }=\leq 1 \\
>10000 \text { inhabitants }\end{array}$ & $\begin{aligned} 10000 \\
0.97\end{aligned}$ & $\begin{aligned} \text { inhabitants) } \\
0.92,1.03\end{aligned}$ & 0.97 & $0.91,1.03$ & 0.93 & $0.88,0.99$ & 0.94 & $0.89,1.00$ & 0.95 & $0.90,1.00$ & 0.97 & $0.92,1.03$ & 0.96 & $0.90,1.01$ & 0.95 & $0.90,1.01$ & 0.99 & $0.94,1.05$ & 0.97 & $0.92,1.03$ \\
\hline
\end{tabular}


Increase in the ratio of consumption to energy intake between 1993 and 2005

\begin{tabular}{|c|c|c|c|c|c|c|c|c|c|}
\hline \multicolumn{10}{|c|}{ Most consumed raw vegetables (mean (sD) intake in 1993 in g) } \\
\hline & $\begin{array}{l}\text { ato } \\
(7.5)) \\
518)\end{array}$ & & $\begin{array}{l}\text { rrot } \\
(5.7)) \\
427)\end{array}$ & & $\begin{array}{l}\text { ilve } \\
(5.5)) \\
882)\end{array}$ & & $\begin{array}{l}\text { mber } \\
(5.8)) \\
305)\end{array}$ & & $\begin{array}{l}\text { ish } \\
4.8) \text { ) } \\
951 \text { ) }\end{array}$ \\
\hline OR $\ddagger$ & $95 \% \mathrm{Cl}$ & OR $\ddagger$ & $95 \% \mathrm{Cl}$ & OR & $95 \% \mathrm{Cl}$ & OR $\ddagger$ & $95 \% \mathrm{Cl}$ & OR $\ddagger$ & $95 \% \mathrm{Cl}$ \\
\hline
\end{tabular}

Area of birth (Ref. $=$ South)

West

North
East

Centre

Dom-Tom

Area of residence $($ Ref. $=$ South

West

North

East

Paris and suburbs

Size of the agglomeration of residence (Ref. $=\begin{array}{lll}0.96 & 0.90,1.03\end{array}$

Quasi-rural (2000-9999 inhabitants) $\quad 0.98 \quad 0.93,1.04$

Quasi-urban (10000-99 999 inhabitants)

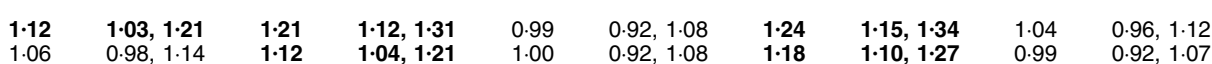

$\begin{array}{llllllllll}1.06 & 0.98,1.14 & \mathbf{1 . 1 2} & \mathbf{1 . 0 4 , 1 . 2 1} & 1.00 & 0.92,1.08 & \mathbf{1 . 1 8} & \mathbf{1 . 1 0 , 1 . 2 7} & 0.99 & 0.92,1.07 \\ 1.04 & 0.96,1.12 & \mathbf{1} .09 & \mathbf{1 . 0 1} \mathbf{1 . 1 7} & 0.96 & 0.89,1.03 & \mathbf{1 . 3 0} & \mathbf{1 . 2 1} 1.40 & 1.05 & 0.98,1.12\end{array}$

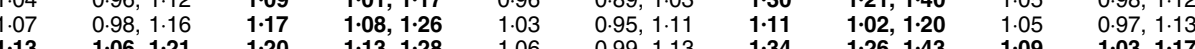

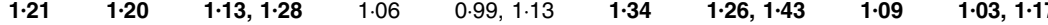

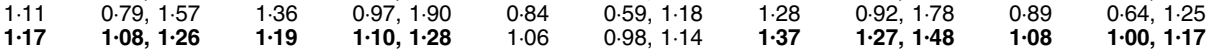

$\begin{array}{lll}0.01 & 0.95,1.08\end{array}$

$0.96,1.06$
0.98

$\geq 0.21$

$0.95 \quad 0.90,1.01$

$\begin{array}{rrrr}\mathbf{1} \cdot 17 & \mathbf{1} \cdot \mathbf{0 8}, \mathbf{1} \cdot \mathbf{2 6} & 1.01 & 0.94,1 \cdot 10\end{array}$

$1.03,1.19 \quad 0.94 \quad 0.87,1.01$

$\begin{array}{lllllll}\mathbf{1} \cdot 03,1 \cdot 19 & 0.96 & 0.90,1.04 & 1.07 & 0.99,1.15 & 1.01 & 0.94,1.08 \\ 0.95,1.12 & 1.02 & 0.94,1.10 & 1.07 & 0.98,1.16 & 1.06 & 0.97 .1 .14\end{array}$

$\begin{array}{llllllll}1.03 & 0.97,1.10 & 0.99 & 0.93,1.05 & \mathbf{1 . 1 4} & \mathbf{1 . 0 7}, \mathbf{1} \cdot \mathbf{2 1} & 0.96 & 0.90,1.02\end{array}$

$\begin{array}{lllllll}0.94,1.05 & 0.98 & 0.93,1.04 & 0.96 & 0.91,1.01 & 1.00 & 0.94,1.05\end{array}$

$\begin{array}{lllllll}0.93,1.04 & 1.00 & 0.95,1.06 & \mathbf{0 . 9 3} & \mathbf{0 . 8 8 , 0 . 9 9} & 1.03 & 0.98,1.09\end{array}$


the recommendations and tended to increase their consumption over the 12-year period, also suggesting a differential impact of the Program in people who were already health-conscious. Those results would be in line with previous results suggesting that national dietary guidelines, when not targeting specific subgroups, reinforce socio-economic inequities $^{(64,65)}$. Alternatively, we can observe that the adoption of healthy dietary patterns became of worldwide concern $^{(66)}$. Awareness about healthy diets and their positive impact emerged among several populations. In a recent publication $^{(67)}$, 'healthy diet' was found to be the first dishchoice motive in a large sample of adults living in France. This may partly explain the overall increase in fruit and vegetable intakes observed in our population. However, it has been shown that this increase was socio-economically patterned. Reduction in health inequities became one of the worldwide priorities only a few years ago ${ }^{(68,69)}$.

We have observed various and complex associations between socio-economic factors and fruit and vegetable subtypes, suggesting that efficient nutritional guidelines cannot be obtained solely by encouraging the consumption of fruits and vegetables as a whole but rather by refining the recommendations according to socio-economic strata. To develop effective nutritional interventions and policies that take the socio-economic environment of individuals into account, future research should focus on understanding (i) the pathways through which population characteristics might influence change in fruit and vegetable consumption as well as (ii) existing interactions between individual and contextual factors. However, we believe that receiving more personalized nutritional guidelines (adapted to the socio-economic profile of the individual) will help contribute to a better understanding and adoption of a healthy diet. Furthermore, beyond quantity, it may be important to promote the variety of fruits and vegetables to ensure nutritional adequacy ${ }^{(70)}$.

\section{Strengths and limitations}

We are aware of some limitations in our study. Some contextual socio-economic data such as access to grocery stores, transportation and neighbourhood safety are not available at the moment in the E3N-EPIC cohort but have been shown to be associated with fruit and vegetable consumption ${ }^{(71,72)}$. Our population study was composed of women only. However, dietary patterns have been shown to be genderpatterned $^{(64)}$. It would therefore be interesting to repeat the analyses among men. Also, women in the E3N-EPIC study tend to have a healthier diet, consume more fruits and vegetables (mean intake of fruits and vegetables being over the recommended 5 servings/ $\mathrm{d}^{(41)}$ ) and are socioeconomically more homogeneous than the general population. However, we were still able to observe associations between socio-economic factors and the evolution of fruit and vegetable consumption in our selected population and we assume that these associations would have been even stronger in the general population where a greater variability in diet and socio-economic environment exists. Also, we were not able to quantify the increase in fruit and vegetable consumption due to the National Nutrition and Health Program ${ }^{(9)}$.

Our study has several strengths. We provided a comprehensive picture of the socio-economic factors associated with an increase in fruit and vegetable consumption over time using dietary data with detailed information on subtypes of fruit and vegetable consumption. Dietary data were collected twice over a 12-year period, in a large sample of women. We studied fruit consumption and vegetable consumption separately, which has been investigated only a few times ${ }^{(18,41,73)}$. Beyond that, to our knowledge, we are the first to study the factors associated with consumption of specific fruits and vegetables. As previously recommended, we were also able to simultaneously consider various socio-economic data including contextual and individual factors $(20,23,63,74)$.

\section{Conclusion}

Our results suggest that some individual, but not contextual socio-economic factors are differentially associated with an increase in fruit or vegetable consumption, or with some fruit and vegetable subtypes. Level of education, number of children, marital and occupational status and level of physical activity were all associated with changes in fruit and vegetable consumption, suggesting that, independently from the environment in which people live, nutritional prevention strategies should focus on actions that have an impact at an individual level (nutrition knowledge, attitudes to healthy eating, food motivation, resources, stressors and psychological resources). Individuals with higher socio-economic position were more likely to increase their fruit and vegetable consumption. It would therefore be important to provide better health interventions in socio-economically disadvantaged groups. Our study also highlights that socioeconomic determinants of fruit and vegetable consumption evolution differ when studying fruit consumption and vegetable consumption separately, as well as according to fruit and vegetable subtypes, underlining the complexity of the relationship between diet and socio-economic environment. This information should be taken into account when developing nutritional prevention strategies to increase fruit and vegetable consumption in the general population. Such diet modifications could have a significant public health impact by decreasing the overall risk of some cancers or other chronic diseases in the general population.

\section{Acknowledgements}

Acknowledgements: The authors are grateful to all participants for providing the data used in the E3N-EPIC study. Financial support: The E3N-EPIC cohort is being carried out with the financial support of the 'Mutuelle Générale de l'Education Nationale' (MGEN); European Community; 
French League against Cancer (LNCC); Gustave Roussy Institute (GR); and French Institute of Health and Medical Research (Inserm). This present work was supported by a grant from the French National Cancer Institute (INCa) and a grant from the World Cancer Research Fund (WCRF; grant number 2015/1389). A.A. is supported by a doctoral grant from INCa. G.F. is supported by the French Research Agency (ANR; Agence Nationale de la Recherche) via an 'Investissement d'Avenir' grant ('Investment for the Future' grant, number ANR-10-COHO-0006) that supports the E4N study. The MGEN, European Community, LNCC, GR, Inserm, INCa, ANR and WCRF had no role in the design, analysis or writing of this article. Conflict of interest: None. Authorship: A.A., F.C.-C., M.-C.B.-R. and G.F. contributed to the data collection. A.A. and G.F. formulated the research questions and designed the research. A.A. performed the statistical analysis and primary manuscript preparation; A.A. and G.F. wrote the paper. G.F. has primary responsibility for the final content; and G.S., C.D., F.R.M., G.R., C.D.P., M.-C.B.-R. and F.C.-C. reviewed the paper and revised it critically. All authors read and approved the final manuscript. Ethics of buman subject participation: This study was conducted according to the guidelines laid down in the Declaration of Helsinki and all procedures involving human subjects were approved by the French National Commission for Computerized Data and Individual Freedom (CNIL). All women signed a letter of informed consent to allow their data to be stored, as required by the CNIL.

\section{Supplementary material}

To view supplementary material for this article, please visit https://doi.org/10.1017/S1368980017003196

\section{References}

1. Tohill BC, Seymour J, Serdula M et al. (2004) What epidemiologic studies tell us about the relationship between fruit and vegetable consumption and body weight. Nutr Rev $\mathbf{6 2}$, 365-374.

2. Hung HC, Joshipura KJ, Jiang R et al. (2004) Fruit and vegetable intake and risk of major chronic disease. $J$ Natl Cancer Inst 96, 1577-1584.

3. Bradbury KE, Appleby PN \& Key TJ (2014) Fruit, vegetable, and fiber intake in relation to cancer risk: findings from the European Prospective Investigation into Cancer and Nutrition (EPIC). Am J Clin Nutr 100, Suppl. 1, 394S-398S.

4. Wang X, Ouyang Y, Liu J et al. (2014) Fruit and vegetable consumption and mortality from all causes, cardiovascular disease, and cancer: systematic review and dose-response meta-analysis of prospective cohort studies. BMJ 349, g4490.

5. Block G, Patterson B \& Subar A (1992) Fruit, vegetables, and cancer prevention: a review of the epidemiological evidence. Nutr Cancer 18, 1-29.

6. World Cancer Research Fund/American Institute for Cancer Research (2007) Food, Nutrition, Physical Activity, and the Prevention of Cancer: A Global Perspective. Washington, DC: AICR.
7. World Health Organization (2003) Diet, Nutrition and the Prevention of Chronic Diseases. Report of the Joint WHO/ FAO Expert Consultation. WHO Technical Report Series no. 916. Geneva: WHO.

8. Direccion Nacional de Promocion de la Salud y Control de Enfermedades no Transmisibles, Ministerio de Salud (2017) Mensajes y gráfica de las Guías Alimentarias para la Población Argentina. http://www.msal.gob.ar/ent/index. php/informacion-para-ciudadanos/menos-sal-vida/482mensajes-y-grafica-de-las-guias-alimentarias-para-la-poblacionargentina (accessed August 2017).

9. Hercberg S, Chat-Yung S \& Chaulia M (2008) The French National Nutrition and Health Program: 2001-2006-2010. Int J Public Health 53, 68-77.

10. National Cancer Institute (2017) 5 A Day for Better Health Program Evaluation Report. Executive Summary. http://cancercontrol.cancer.gov/5ad_exec.html (accessed August 2017).

11. NHS Choices (2017) 5 A DAY: what counts? http://www. nhs.uk/Livewell/5ADAY/Pages/Whatcounts.aspx (accessed August 2017)

12. Giskes K, Avendano M, Brug J et al. (2010) A systematic review of studies on socioeconomic inequalities in dietary intakes associated with weight gain and overweight/obesity conducted among European adults. Obes Rev 11, 413-429.

13. Hare-Bruun H, Togo P, Andersen LB et al. (2011) Adult food intake patterns are related to adult and childhood socioeconomic status. J Nutr 141, 928-934.

14. Leech RM, McNaughton SA \& Timperio A (2014) The clustering of diet, physical activity and sedentary behavior in children and adolescents: a review. Int J Behav Nutr Phys Act 11, 4 .

15. Pechey R, Jebb SA, Kelly MP et al. (2013) Socioeconomic differences in purchases of more vs. less healthy foods and beverages: analysis of over 25,000 British households in 2010. Soc Sci Med $92,22-26$.

16. Whichelow MJ \& Prevost AT (1996) Dietary patterns and their associations with demographic, lifestyle and health variables in a random sample of British adults. Br J Nutr $\mathbf{7 6}$, $17-30$.

17. Irala-Estevez JD, Groth M, Johansson L et al. (2000) A systematic review of socio-economic differences in food habits in Europe: consumption of fruit and vegetables. Eur J Clin Nutr 54, 706-714.

18. Kamphuis CB, Giskes K, de Bruijn GJ et al. (2006) Environmental determinants of fruit and vegetable consumption among adults: a systematic review. Br J Nutr 96, 620-635.

19. Pechey R, Monsivais P, Ng YL et al. (2015) Why don't poor men eat fruit? Socioeconomic differences in motivations for fruit consumption. Appetite 84, 271-279.

20. Braveman PA, Cubbin C, Egerter S et al. (2005) Socioeconomic status in health research: one size does not fit all. JAMA 294, 2879-2888.

21. Lassale C, Galan P, Castetbon K et al. (2013) Differential association between adherence to nutritional recommendations and body weight status across educational levels: a cross-sectional study. Prev Med 57, 488-493.

22. Wyndels K, Dallongeville J, Simon C et al. (2011) Regional factors interact with educational and income tax levels to influence food intake in France. Eur J Clin Nutr 65, 1067-1075.

23. Turrell G, Hewitt B, Patterson C et al. (2003) Measuring socio-economic position in dietary research: is choice of socio-economic indicator important? Public Health Nutr 6 , 191-200.

24. Liberatos P, Link BG \& Kelsey JL (1988) The measurement of social class in epidemiology. Epidemiol Rev 10, 87-121.

25. Darmon N \& Drewnowski A (2008) Does social class predict diet quality? Am J Clin Nutr 87, 1107-1117.

26. Bazzano LA, He J, Ogden LG et al. (2002) Fruit and vegetable intake and risk of cardiovascular disease in US adults: 
the first National Health and Nutrition Examination Survey Epidemiologic Follow-up Study. Am J Clin Nutr 76, 93-99.

27. Boffetta P, Couto E, Wichmann J et al. (2010) Fruit and vegetable intake and overall cancer risk in the European Prospective Investigation into Cancer and Nutrition (EPIC). J Natl Cancer Inst 102, 529-537.

28. Harding AH, Wareham NJ, Bingham SA et al. (2008) Plasma vitamin $\mathrm{C}$ level, fruit and vegetable consumption, and the risk of new-onset type 2 diabetes mellitus: the European prospective investigation of cancer-Norfolk prospective study. Arch Intern Med 168, 1493-1499.

29. Ledoux TA, Hingle MD \& Baranowski T (2011) Relationship of fruit and vegetable intake with adiposity: a systematic review. Obes Rev 12, e143-e150.

30. Liu RH (2003) Health benefits of fruit and vegetables are from additive and synergistic combinations of phytochemicals. Am J Clin Nutr 78, 3 Suppl, 517S-520S.

31. Van Duyn MA \& Pivonka E (2000) Overview of the health benefits of fruit and vegetable consumption for the dietetics professional: selected literature. J Am Diet Assoc 100, 1511-1521.

32. Goldberg JP (1992) Nutrition and health communication: the message and the media over half a century. Nutr Rev $\mathbf{5 0}$, 71-77.

33. Wills JM, Storcksdieck genannt BS, Kolka M et al. (2012) European consumers and health claims: attitudes, understanding and purchasing behaviour. Proc Nutr SOC 71, 229-236.

34. Clavel-Chapelon F, van Liere MJ, Giubout C et al. (1997) E3N, a French cohort study on cancer risk factors. E3N Group. Etude Epidemiologique aupres de femmes de l'Education Nationale. Eur J Cancer Prev 6, 473-478.

35. Riboli E \& Kaaks R (1997) The EPIC Project: rationale and study design. European Prospective Investigation into Cancer and Nutrition. Int J Epidemiol 26, Suppl. 1, S6-S14.

36. Cottet V, Touvier M, Fournier A et al. (2009) Postmenopausal breast cancer risk and dietary patterns in the E3N-EPIC prospective cohort study. Am J Epidemiol 170, $1257-1267$.

37. van Liere MJ, Lucas F, Clavel F et al. (1997) Relative validity and reproducibility of a French dietary history questionnaire. Int J Epidemiol 26, Suppl. 1, S128-S136.

38. Estaquio C, Kesse-Guyot E, Deschamps V et al. (2009) Adherence to the French Programme National Nutrition Sante Guideline Score is associated with better nutrient intake and nutritional status. J Am Diet Assoc 109, 1031-1041.

39. Affret A, Severi G, Dow C et al. (2017) Socio-economic factors associated with a healthy diet: results from the E3N study. Public Health Nutr 20, 1574-1583.

40. Institut de veille sanitaire (2006) Etude nationale nutrition santé ENNS. http://opac.invs.sante.fr/doc_num.php?explnum_id=3481 (accessed November 2017).

41. Estaquio C, Druesne-Pecollo N, Latino-Martel $\mathrm{P}$ et al. (2008) Socioeconomic differences in fruit and vegetable consumption among middle-aged French adults: adherence to the 5 A Day recommendation. J Am Diet Assoc 108, 2021-2030.

42. AFSSA (Agence Française de Sécurité Sanitaire des Aliments) (2009) INCA2 - Etude Individuelle Nationale des Consommations Alimentaires 2. https://www.anses.fr/fr/system/ files/PASER-Ra-INCA2.pdf (accessed November 2017).

43. Buckland G, Ros MM, Roswall N et al. (2014) Adherence to the Mediterranean diet and risk of bladder cancer in the EPIC cohort study. Int J Cancer 134, 2504-2511.

44. Buckland G, Agudo A, Lujan L et al. (2010) Adherence to a Mediterranean diet and risk of gastric adenocarcinoma within the European Prospective Investigation into Cancer and Nutrition (EPIC) cohort study. Am J Clin Nutr 91, 381-390.
45. Rey G, Jougla E, Fouillet A et al. (2009) Ecological association between a deprivation index and mortality in France over the period 1997-2001: variations with spatial scale, degree of urbanicity, age, gender and cause of death. BMC Public Health 9, 33.

46. INSEE (Institut National de la Statistique et des Etudes Economiques) (n.d.) 1999 population census data; available at https://www.insee.fr/fr/statistiques/2414782?sommaire $=2414232$. Tax authority's 2001 household income data; available at https://www.insee.fr/fr/statistiques/1893279 (accessed August 2017).

47. Bertin M, Touvier M, Dubuisson C et al. (2016) Dietary patterns of French adults: associations with demographic, socio-economic and behavioural factors. J Hum Nutr Diet 29, 241-254.

48. Macintyre S (2007) Deprivation amplification revisited; or, is it always true that poorer places have poorer access to resources for healthy diets and physical activity? Int J Behav Nutr Phys Act 4, 32.

49. Perrin AE, Dallongeville J, Ducimetiere P et al. (2005) Interactions between traditional regional determinants and socio-economic status on dietary patterns in a sample of French men. Br J Nutr 93, 109-114.

50. Beenackers MA, Kamphuis CB, Giskes K et al. (2012) Socioeconomic inequalities in occupational, leisure-time, and transport related physical activity among European adults: a systematic review. Int J Behav Nutr Phys Act 9, 116.

51. Cameron AJ, Spence AC, Laws R et al. (2015) A review of the relationship between socioeconomic position and the early-life predictors of obesity. Curr Obes Rep 4, 350-362.

52. Hiscock R, Bauld L, Amos A et al. (2012) Socioeconomic status and smoking: a review. Ann NY Acad Sci 1248, $107-123$.

53. Mackenbach JD, Brage S, Forouhi NG et al. (2015) Does the importance of dietary costs for fruit and vegetable intake vary by socioeconomic position? Br J Nutr 114, 1464-1470.

54. Dubowitz T, Heron M, Bird CE et al. (2008) Neighborhood socioeconomic status and fruit and vegetable intake among whites, blacks, and Mexican Americans in the United States. Am J Clin Nutr 87, 1883-1891.

55. Johansson L, Thelle DS, Solvoll K et al. (1999) Healthy dietary habits in relation to social determinants and lifestyle factors. Br J Nutr 81, 211-220.

56. Mackenbach JD, Burgoine T, Lakerveld J et al. (2017) Accessibility and affordability of supermarkets: associations with the DASH diet. Am J Prev Med 53, 55-62.

57. Shohaimi S, Welch A, Bingham S et al. (2004) Residential area deprivation predicts fruit and vegetable consumption independently of individual educational level and occupational social class: a cross sectional population study in the Norfolk cohort of the European Prospective Investigation into Cancer (EPIC-Norfolk). J Epidemiol Community Health 58, 686-691.

58. Ball K, Crawford D \& Mishra G (2006) Socio-economic inequalities in women's fruit and vegetable intakes: a multilevel study of individual, social and environmental mediators. Public Health Nutr 9, 623-630.

59. Giskes K, van Lenthe FJ, Kamphuis CB et al. (2009) Household and food shopping environments: do they play a role in socioeconomic inequalities in fruit and vegetable consumption? A multilevel study among Dutch adults. J Epidemiol Community Health 63, 113-120.

60. Aggarwal A, Cook AJ, Jiao J et al. (2014) Access to supermarkets and fruit and vegetable consumption. Am J Public Health 104, 917-923.

61. Keane A, Cullinan J, Perry CP et al. (2016) Dietary quality in children and the role of the local food environment. SSM Popul Health 2, 770-777. 
62. Roos G, Johansson L, Kasmel A et al. (2001) Disparities in vegetable and fruit consumption: European cases from the north to the south. Public Health Nutr 4, 35-43.

63. Galobardes B, Morabia A \& Bernstein MS (2001) Diet and socioeconomic position: does the use of different indicators matter? Int J Epidemiol 30, 334-340.

64. Sorensen G, Stoddard AM, Dubowitz T et al. (2007) The influence of social context on changes in fruit and vegetable consumption: results of the healthy directions studies. $\mathrm{Am} \mathrm{J}$ Public Health 97, 1216-1227.

65. Stables GJ, Subar AF, Patterson BH et al. (2002) Changes in vegetable and fruit consumption and awareness among US adults: results of the 1991 and 19975 A Day for Better Health Program surveys. J Am Diet Assoc 102, 809-817.

66. World Health Organization (2004) Global Strategy on Diet, Physical Activity and Health. Geneva: WHO.

67. Ducrot P, Mejean C, Alles B et al. (2015) Motives for dish choices during home meal preparation: results from a large sample of the NutriNet-Sante study. Int J Behav Nutr Phys Act 12, 120.

68. Stronks K, Toebes B, Hendriks A et al. (2016) Social Justice and Human Rights as a Framework for Addressing Social Determinants of Health. Final Report of the Task Group on Equity, Equality and Human Rights. Review of Social
Determinants of Health and the Health Divide in the WHO European Region. Copenhagen: WHO Regional Office for Europe.

69. World Health Organization (2011) Promoting Health and Reducing Health Inequities by Addressing the Social Determinants of Health. Copenhagen: WHO Regional Office for Europe.

70. Giskes K, Turrell G, Patterson C et al. (2002) Socioeconomic differences among Australian adults in consumption of fruit and vegetables and intakes of vitamins A, C and folate. J Hum Nutr Diet 15, 375-385.

71. Caspi CE, Sorensen G, Subramanian SV et al. (2012) The local food environment and diet: a systematic review. Health Place 18, 1172-1187.

72. Robinson PL, Dominguez F, Teklehaimanot S et al. (2013) Does distance decay modelling of supermarket accessibility predict fruit and vegetable intake by individuals in a large metropolitan area? J Health Care Poor Underserved 24, 172-185.

73. Trudeau E, Kristal AR, Li S et al. (1998) Demographic and psychosocial predictors of fruit and vegetable intakes differ: implications for dietary interventions. J Am Diet Assoc 98, 1412-1417.

74. Lallukka T, Laaksonen M, Rahkonen O et al. (2007) Multiple socio-economic circumstances and healthy food habits. Eur J Clin Nutr 61, 701-710. 